\title{
Lenguaje claro en el siglo XXI: panorama general y propuesta de aplicación al proceso de traducción y corrección
}

\author{
ROMINA MARAZZATO SPARANO \\ University at Buffalo y Plain Language International Inc. \\ romina@languagecompass.com
}

\section{Resumen}

En este artículo se presenta un panorama del lenguaje claro en tanto enfoque para la redacción y en tanto movimiento alineado con el derecho a entender como derecho civil, y se explora su rol en textos informativos de contenido, con particular interés en su aplicación a la traducción y la corrección. Se parte de una concepción funcional del texto y multifacética del proceso de redacción, traducción y corrección que tiene en cuenta las normas internacionales de ISO y ASTM. Primeramente, se aborda la definición de lenguaje claro elaborada en el seno de la Federación Internacional de Lenguaje Claro (IPLF) y adoptada como punto de inicio en el proyecto normativo ISO 24495 sobre lenguaje claro (aún en desarrollo). También, se ofrece un panorama histórico de los hitos del lenguaje claro como movimiento iniciado en el siglo XX en el contexto internacional y en el mundo hispanohablante. Seguidamente, se enumeran principios clave del lenguaje claro que surgen del juicio de expertos e incluyen pertinencia, accesibilidad, inteligibilidad y aplicabilidad; se sugiere desdoblar el principio de inteligibilidad en textualidad y adecuación para facilitar una aproximación escalonada a posibles intervenciones. Se toman nociones de lingüística funcional para definir texto y presentar técnicas de expresión que promuevan la claridad. Se proponen técnicas para la solución de problemas de inteligibilidad, tanto en la redacción de texto monolingüe (de partida o de llegada) como en el proceso de traducción, ya que estas instancias atañen específicamente a la tarea del traductor y del corrector.

\section{Palabras clave}

Lenguaje claro, traducción, corrección, textualidad, adecuación. 


\title{
Plain Language in the 21st Century: Overview and Application Proposal to the Translation and Editing Process
}

\begin{abstract}
We present here an overview of plain language as an approach to writing and as a movement aligned with the right to understand as a civil right, and explore its role in informative texts, with particular interest in its application to translation and editing. We start from a functional and multifaceted conceptualization of the writing, translation and editing processes, reflected in ISO and ASTM standards. First, we present the definition of plain language developed within the International Plain Language Federation (IPLF) and adopted as a starting point for the development of ISO Standard 24495 on plain language (still underway). We also offer a historical overview of the milestones of plain language as a movement started in the 20th century-both internationally and in the Spanish-speaking world. Next, we present key principles of plain language as outlined by experts, including relevance, accessibility, intelligibility, and applicability; we suggest the principle of intelligibility be broken down into textuality and adequacy to enable a stepwise approach to text revisions. Finally, in considering the notion of text, we borrow from functional linguistics to contextualize the strategies presented to promote clarity. We focus on strategies to solve intelligibility problems, both in the writing and editing of monolingual text (source or target) and in translation, as these tasks specifically pertain to the work of translators and editors.
\end{abstract}

\section{Keywords}

Plain language, translation, editing, textuality, adequacy.

Recibido el 03/05/2021

Aceptado el 07/07/2021 


\section{Introducción}

En el presente artículo se ofrece un panorama del lenguaje claro en tanto enfoque para la redacción y en tanto movimiento alineado con el derecho a entender como derecho civil, y se explora su rol en textos informativos de contenido científico-técnico, con particular interés en su aplicación a la traducción y la corrección de textos.

Primeramente, se presenta una definición del lenguaje claro, tomada como base para el desarrollo de una nueva norma ISO al respecto, y una breve reseña del movimiento homónimo iniciado en el siglo XX, así como de las iniciativas que se encuentran en desarrollo. A continuación, se detallan principios clave de redacción en lenguaje claro, establecidos a partir del juicio de expertos, y se proponen una serie de estrategias para abordar aspectos inherentes a la inteligibilidad de un texto monolingüe y a la traducción.

Previamente, conviene precisar la perspectiva desde la que se abordan la redacción, la traducción y la corrección de textos. Para empezar, se toma la noción de texto de la lingüística funcional, originada en el trabajo de Halliday y Hasan (1976), que considera el texto como una unidad semántica que "crea continuamente su propia cohesión" y se relaciona "de forma relativamente estable y coherente con los contextos en los que funciona para significar" (Eggins, 2011: 53, traducción de la autora). Esta perspectiva permite combinar elementos de gramática clásica, como la noción de clase de palabras (sustantivo, verbo, preposición, etc.), con nociones aportadas por escuelas del siglo XX (Ghio y Fernández, 2008), como la distinción entre tema y rema proveniente de la Escuela de Praga (a grandes rasgos, el punto de partida temático de la oración y su elucidación o elaboración; no se profundiza aquí sobre la distinción entre posición inicial o tópico y base argumental o tema, véase LaPolla, 2009) y la interacción entre las nociones discursivas de patrones organizativos y superestructuras desarrolladas por Horowitz (1985) y Van Dijk (1980) (Bratcher Hoskins, 1986).

En este contexto, se entiende la redacción como un proceso complejo, no lineal sino recursivo, alimentado por una red dinámica de asociaciones entre las etapas de la propuesta clásica de Hayes y Flower (1980) de planificación, textualización y revisión, sobre el que se ha teorizado desde diversas perspectivas (Laggette et al. 2019). En la industria lingüística (que incluye el mundo editorial monolingüe y el mundo de la traducción), a la redacción de un escrito original se agrega su corrección y, posiblemente, su traducción. La primera puede incluir la corrección conceptual, de estilo y ortotipográfica (Marazzato Sparano y Martin, 2018) para llegar al texto final susceptible de publicación, que aún debe someterse a una verificación de pruebas monolingüe (Martínez de Sousa, 1999), incluso en contextos digitales.

Tradicionalmente, una vez finalizado ese proceso, se procede a la traducción del texto a otros idiomas, entendida esta como la transferencia de un idioma a otro de manera que "el contenido y, en muchos casos, la forma de ambos textos pueda considerarse equivalente" (ASTM, 2014). En el siglo XXI, con la expansión del texto digital, incluso como parte de la interfaz de aplicaciones, la traducción suele comenzar antes de la finalización del texto original y verse inmersa en el proceso de globalización, 
internacionalización, localización y traducción (GIL ${ }^{1}$ ) que, cada vez más, se desarrolla en paralelo a la producción del texto de partida (Maylath y Amant, 2019). Por último, el texto traducido también ha de pasar por etapas de corrección, particularmente por una etapa de corrección bilingüe (ASTM, 2014 e ISO, 2015), para cotejar la correspondencia del texto de llegada con el texto de partida.

A lo largo de este trabajo, se emplean ejemplos provenientes de un corpus compilado por la autora a lo largo de su trayectoria profesional, principalmente circunscrito a temas de salud. Por motivos de confidencialidad, no se comparte la proveniencia de los ejemplos particulares. Los ejemplos incluyen fragmentos en idioma original, fragmentos traducidos y fragmentos en distintas etapas de revisión. Como es habitual en el abordaje de cuestiones de revisión, se indican las instancias problemáticas con un signo de interrogación (?) y las instancias erróneas con un asterisco ( ${ }^{*}$ ).

\section{Definición}

Según la Federación Internacional del Lenguaje Claro (conformada por las asociaciones internacionales de lenguaje claro), un escrito "está en lenguaje claro si su redacción, estructura y diseño son tan transparentes que los lectores a los que se dirige pueden encontrar lo que necesitan, entender lo que encuentran y usar esa información" (IPLF, s. f.).

Esta definición surge del trabajo de expertos que hoy forman parte de las asociaciones internacionales de lenguaje claro (sobre las se brindan más detalles en la sección siguiente), como, entre muchos otros, la doctora Annetta Cheek (2010: 5-15), que lideró la formación del Center for Plain Language y el proyecto de Ley de Escritura Clara en EE. UU. sancionada en 2010 (Plain Writing Act, 2010); Joseph Kimble, profesor de derecho y miembro fundador de CPL (1992, 1996-1997); Cheryl Stephens (2007, 2010: 12-17) y Kate Harrison-Whiteside (2007), canadienses, fundadoras de la asociación Plain Language Association International; y Julie Clement, jefa de redacción de la revista The Clarity Journal durante trece años y actual presidenta de Clarity International.

Cabe notar que esta definición adopta una noción intuitiva de claridad que conviene explicitar brevemente. Se toma aquí la noción de claridad desde una perspectiva funcional, en la que la claridad es la construcción de sentido mediante la elección de opciones de redacción que combinen la mayor precisión, concisión y fluidez posible de cara al fin comunicativo. Autores como Gutiérrez Rodilla (2005) y Giba (2014) señalan que precisión, concisión (o economía en Gutiérrez Rodilla) y fluidez son características esenciales de la claridad, tanto en textos especializados como legos (Gutiérrez Rodilla, 2005: 20). Giba (2014: 17) apunta, como lo han hecho muchos otros autores, que la claridad en la redacción refleja la claridad de pensamiento. Estos autores entienden la claridad como una cualidad dinámica del texto que requiere de la intervención del autor y de la connivencia del lector para lograr la máxima comprensión posible en el menor

\footnotetext{
1 Durante el proceso GILT, se adaptan diversos aspectos de la elaboración de productos o servicios digitales destinados a mercados específicos (Kelly, 2020) que incluyen la infraestructura o marco de trabajo (globalización), el código (internacionalización), la interfaz o experiencia que se le ofrece al usuario (localización) y el mensaje lingüístico en sí mismo (traducción).
} 
tiempo posible. Para redactar con claridad, el autor ha de evitar la ambigüedad, hacer que cada palabra cuente y procurar que cada frase conduzca coherente y elegantemente a la siguiente. Asimismo, el lector debe aportar su atención, intención y bagaje de conocimiento previo. Ahora bien, como indica Williams (1990: x) "el placer estético y la claridad no son en absoluto excluyentes; de hecho, suelen formar parte de la misma experiencia" (traducción de la autora).

En contraste, un texto oscuro es un texto mal escrito, que resulta difícil de leer, ambiguo o, incluso, incomprensible para su lector meta. Sin duda, un texto que trata de una materia especializada puede resultar confuso a lectores no especializados en ella, pero estos no son sus lectores meta. La oscuridad, en cambio, vuelve confuso un texto destinado a su público meta y puede surgir por diferentes razones (Williams, 1990: 19): ambigüedad, falta de conocimiento o confusión conceptual sobre el tema tratado, descuido o falta de tiempo para revisar el texto, malos hábitos o pautas de redacción, incompetencia o falta de práctica, incapacidad inconsciente para reconocer un conocimiento previo no compartido por el lector, o, incluso, como una estrategia deliberada para evitar críticas y ganar adeptos "faltos de autoestima” (Krashen, 2012). Cabe subrayar, sin embargo, que la oscuridad no es sinónimo de complejidad. Un texto complejo puede reflejar con claridad ideas complejas (Williams, ibid.: xi).

La claridad resulta indispensable en textos que requieren de la capacidad del lector para entender, evaluar y utilizar información a fin de enfrentar los retos de la sociedad moderna y obtener resultados positivos a nivel personal, comunitario, regional y global (OECD, 2016: 38). Se llama aquí textos informativos a aquellos que apuntan a que el lector entienda o aprenda un concepto, adquiera una habilidad, tome una decisión o lleve a cabo una tarea o proceso. Ciertamente, la noción de tipo textual es compleja, con propuestas clasificatorias diversas y una terminología no necesariamente cristalizada (Ciapuscio, 2005); por ejemplo, "los tipos textuales se confunden, a veces, con el concepto de género" (Sánchez Trigo, 2002: 124).

Se entiende aquí el texto informativo como una categoría amplia que utiliza diversas superestructuras y patrones organizativos (en el sentido señalado por Bratcher Hoskins, 1986) y, sobre todo, hace hincapié en la función referencial del lenguaje. Desde la perspectiva jakobsoniana (Jakobson, 1960), esta función se basa en la capacidad del lenguaje de remitir a la realidad, designarla o representarla. El texto informativo se construye a partir de la selección de contenido fáctico y puede incluir secuencias de carácter espacial, temporal, lógico, persuasivo o procedural, asociadas a la clasificación de bases textuales - descriptiva, narrativa, expositiva, argumentativa e instructiva- propuestas en la década de 1970 por autores como Werlich y Adam (Ciapuscio, 2005). Asimismo, un texto informativo puede adscribir a diversos géneros desde el punto de vista bajtiniano de género como constructo semiótico-social (Bajtin, 1999). Es decir, el texto informativo se actualiza en una variedad de géneros, más allá de los característicos del discurso científico-técnico (artículos, ensayos, informes, etc.) usualmente asociados a la transmisión de conocimiento (Gutiérrez Rodilla, 2005: 9). Por ejemplo, durante la pandemia de COVID-19, se han publicado libros de cuentos infantiles para ayudar a padres y niños a afrontar la crisis multifacética generada por la enfermedad y las medidas concomitantes (véase Healthwise, 2020). Por otro lado, se ha 
de subrayar que, al concentrarse en un factor comunicativo y redactar así un tipo de texto, no se pueden desatender los demás, sino que se ha de abogar por el equilibrio en el tratamiento de los diversos factores comunicativos como andamiaje esencial para la efectividad del texto.

\subsection{Breve observación sobre fórmulas de legibilidad}

Las discusiones sobre lenguaje claro concitan a menudo la mención de fórmulas de legibilidad (Schriver, 2017: 12). Rudolph Flesch, pionero del lenguaje claro en Estados Unidos, del que se hablará en la sección siguiente, desarrolló una de las primeras fórmulas de legibilidad para seleccionar textos adecuados a distintas necesidades educativas, particularmente de adultos. La fórmula de Flesch (1948) atiende, en una primera instancia, al promedio de palabras por oración y de sílabas por palabra (ver figura 1) y, en una segunda instancia, al uso de palabras de "interés humano", como pronombres personales, palabras que denotan género y uso de diálogo, preguntas y exclamaciones.

$$
206,835-\left(1,015 \times \frac{\text { total de palabras }}{\text { total de oraciones }}\right)-\left(84,6 \times \frac{\text { total de sílabas }}{\text { total de palabras }}\right)
$$

Figura 1. Primera parte de la fórmula de Flesch, que mide la facilidad de lectura y arroja un puntaje de cero a cien, en una escala de difícil (0) a fácil (100).

Interés humano $=3,635 p p+0,314$ op

pp: porcentaje de palabras personales

op: porcentaje de oraciones personales

Figura 2. Segunda parte de la fórmula de Flesch, que mide el "interés humano" y arroja un puntaje de cero a cien, en una escala de 0 (aburrido o muy técnico a 100 - drama o ficción-), considerándose un puntaje de 40-60 como "muy interesante"

Además de la fórmula de Flesch, se han desarrollado diversas propuestas y reformulaciones de las fórmulas tanto en inglés (Scott, s. f.) como en español (Muñoz Fernández, 2018) y otros idiomas, siempre sobre la base del conteo de sílabas, palabras y oraciones, y sin reconocer que "un buen puntaje [de legibilidad] no es garantía de una buena redacción" (Kearl, 1948). En usos posteriores de la fórmula de Flesch, se abandonan completamente "las sutilezas de los hallazgos de Flesch" (Vieth, 1988: 69) y se deja de atender al contenido mismo (la personalización en textos de divulgación y la relevancia y el rigor en textos especializados) y a los aspectos formales del texto que contribuyen a su legibilidad.

Una de las más empleadas en la actualidad es la fórmula de Flesch-Kincaid (Kincaid, 1975), que, a partir de la fórmula de Flesch, arroja un nivel de dificultad del texto según el grado de escolarización de quien pueda leerlo con facilidad (ver figura 3). Hoy en día, además de las limitaciones que ya explicitaban Flesch y sus contemporáneos (Vieth, 
1988: 60-66), las fórmulas resultan inestables en sus versiones digitales, pues, según el software empleado o la preparación del texto, incluso la misma fórmula puede arrojar resultados diferentes sobre el mismo texto (Schriver, 2017). A menudo, para ser procesado por una fórmula, un documento se debe despojar de características esenciales del lenguaje claro, como el uso de recursos ortotipográficos y de diseño, que aumentan la comprensibilidad. Shriver, experta en el campo del diseño para la comunicación, hace particular hincapié en la importancia de este aspecto, al que se aboca detalladamente en su difundido libro sobre el tema, Dynamics in Document Design (La dinámica del diseño de documentos, 1996).

$$
0.39-\left(1,015 \times \frac{\text { total de palabras }}{\text { total de oraciones }}\right)+\left(11,8 \times \frac{\text { total de sílabas }}{\text { total de palabras }}\right)-15.59
$$

Figura 3. Fórmula de nivel de grado de Flesch-Kincaid, cuyo resultado indica el nivel escolar de quien pueda leer el texto evaluado facilidad.

Así pues, los profesionales del lenguaje claro no recomiendan el uso de fórmulas de legibilidad como baremo de la calidad o comprensibilidad de un texto (Jarret y Ridish, 2019). Sin embargo, las fórmulas aún se utilizan en procesadores de textos como MS Word, se aplican a la evaluación de textos a nivel gubernamental (por ejemplo, en el Departamento de Defensa de EE. UU.) y forman parte de los algoritmos SEO de posicionamiento en buscadores, lo cual quizás deba llamar a reflexión.

El uso de fórmulas de legibilidad, además, ha dado apoyo a extrapolaciones que deben ser exploradas. Por ejemplo, dado que las palabras y oraciones breves obtienen un mejor puntaje al ser evaluadas por las fórmulas, se suele aconsejar, sin morigeración, el uso de palabras y oraciones cortas. Esto lleva a que se omitan conectores y marcadores discursivos que, al combinar cláusulas, generan inevitablemente oraciones más largas y no por ello menos comprensibles; de hecho, estudios sobre la reparación de relaciones lógicas mediante la introducción de marcadores indican que tanto los lectores más avanzados como los menos expertos se benefician $=$ (Linderholm et al., 2000).

En una línea de argumentación similar, se arguye también que estructuras sintácticas que reclaman mayor actividad neuronal o tiempo de respuesta en estudios de resonancia magnética funcional, como es el caso de la voz pasiva (por ej., Mack, 2013) o las subordinadas (por ej., Vogelzang et al., 2020), deben evitarse a fin de aligerar la carga cognitiva del lector. Sin embargo, los estudios de comprensión lectora al respecto, que aún deben fortalecerse, no necesariamente apoyan esta conclusión. Benjamin Bergen y Nancy Chang (en preparación), del Laboratorio de Ciencias Cognitivas de University of California San Diego, señalan que la voz activa "lleva al comprensor a simular un evento desde la perspectiva perceptiva o motora del agente, mientras que la voz pasiva podría llevar a la simulación desde la perspectiva del paciente" (traducción de la autora) y esto tiene efectos en la empatía con los diversos participantes del evento.

Este tipo de cuestiones tiene que ver con la identidad de la lengua escrita que - a pesar de mantener una estrecha relación con la lengua oral- hace un uso idiosincrático de formas y estructuras tendientes a una sofisticada construcción y divulgación del 
conocimiento (Piacente, 2014). La ensayista argentina Beatriz Sarlo señala al respecto que la lengua escrita conserva y aporta niveles retóricos esenciales a la comunicación como "el sistema de subordinación [y] frases complejas, etc. que son fundamentales para pensar. Sin subordinar, sin subordinadas, no se puede pensar, sin pese a esto, sin embargo, no obstante, sin eso, no se puede pensar".

\section{Antecedentes del movimiento de lenguaje claro}

El lenguaje claro como movimiento surge en el siglo XX como respuesta a las jergas burocrática, legal y técnica, tanto en el ámbito gubernamental como privado, a fin de brindar información y acceso a servicios al ciudadano y de responder a las necesidades y demandas del consumidor. Hoy en día, también apunta a zanjar brechas en las llamadas alfabetizaciones específicas (médica, financiera, digital, etc.), así como a promover el desarrollo científico interdisciplinar y su divulgación (Marazzato Sparano, 2017, 2019). La comunicación clara se hace especialmente necesaria en el accionar político, legislativo y educativo tendente a promover el bienestar individual, social y medioambiental, y en la práctica científica, donde el imperativo de "publicar o perecer" ha contribuido a la propagación de ideas fraudulentas, obsoletas o erróneas (Krashen, 2012).

A lo largo de la historia, ha habido diversos llamados a un lenguaje llano (Garner, 2009: 40-41). En el primer siglo de la era común, Quintiliano, quien, según historiadores como Alfonso Reyes (2018), establece un programa didáctico que sienta las bases de la educación liberal actual, llama no solo a promover la comprensión sino a que "de ninguna manera se cuele el malentendido" en su obra Instituciones Oratorias (8.2.24). En el siglo XIII, el rey Alfonso X el Sabio hizo redactar en castellano y no en latín, como hasta entonces, todos los documentos oficiales de uso interno, no solo por razones personales y políticas (su ascendencia castellana) sino también para apoyar la comprensión, ya que "Castilla era el reino con más peso demográfico, de mayor extensión territorial y con una economía más pujante” (Fernández-Ordóñez, s.f.), donde, además, el español se difundió con los cantares de gesta, que servían de ejemplo e inspiración, lo mismo que las historias de los superhéroes de hoy (Marazzato Sparano, 2016). En el siglo XV, el rey Enrique V de Inglaterra alentó el uso del idioma inglés, hablado por el pueblo, en la documentación oficial y la literatura, oponiéndose al dominio previo del idioma francés (Cartwright, 2020).

\subsection{El lenguaje claro a nivel internacional}

En su versión contemporánea, el concepto de lenguaje claro proviene del mundo angloparlante, con propuestas iniciadas en la primera mitad del siglo XX, como la de Rudolph Flesch en los años 1940, delineada en su tesis doctoral Marks of a Readable Style (Marcas de un estilo legible, de 1943, citada en Flesch, 1948) y en libros de divulgación como The Art of Readable Writing (El arte de la escritura legible, 1949) y The Art of Clear Thinking (El arte de pensar claro, 1951). Flesch trabaja originalmente con textos para adultos sin altos niveles de alfabetización a fin de promover su 
capacitación en un mundo cada vez más especializado. El interés de Flesch en la capacitación se enmarca en un periodo que se enfrentó al crecimiento exponencial del número de alumnos en escuelas primarias y secundarias para el que maestros, profesores y administradores estaban poco preparados (Murphy, 2012: 235).

Otros dos hitos del siglo XX incluyen el llamado de Maury Maverick (1944) y de John O'Hayre (1966), ambos funcionarios, a renunciar al galimatías del lenguaje burocrático en el seno del gobierno. Maverick redactó un famoso comunicado oficial durante la Segunda Guerra Mundial y O’Hayre publicó un manual de estilo sobre lenguaje llano.

Ya en la segunda mitad del siglo XX, y en los ámbitos empresarial y gubernamental, dos hitos impulsaron la adopción del lenguaje claro. El primero fue la intervención de Bess Myerson, exmiss Estados Unidos y famosa defensora de los derechos del consumidor, en la reformulación de textos financieros a principios de la década de 1970, que llevó a la creación del primer contrato en lenguaje claro en el sector financiero (desarrollado tanto en inglés como en español) en Citibank (MacDonald, 2015).

El segundo hito surge en el Reino Unido con Chrissie Maher, defensora de los derechos del ciudadano, quien identificó el fatal alcance de un confuso formulario de instrucciones que llevó a la muerte de dos mujeres por no haber logrado obtener ayuda para pagar sus facturas de calefacción (Maher, 1998). Maher fundó en 1979, junto a Martin Cutts, Plain English Campaign (http://www.plainenglish.co.uk/), una empresa de servicios para promover cambios legislativos. Por diversos motivos, los destinos de estos dos fundadores se bifurcaron, pero ambos continuaron trabajando por el inglés claro: Maher en Plain English Campaign y Cutts en Plain English Commission (que fundó en 1989), hoy Plain Language Commission (https://www.clearest.co.uk/), con la publicación de la Oxford Guide to Plain English (2013), ya en su cuarta edición.

Estos hitos contribuyeron a impulsar la formación de redes de lenguaje claro en el ámbito gubernamental y la fundación de asociaciones en el ámbito profesional. En el sector público, se promovió legislación sobre el uso de lenguaje claro en la redacción de nuevos documentos y la reforma de procesos y documentos existentes (para un cronograma de acontecimientos en EE. UU., véase Schriver, 2017). El proceso legislativo tomó impulso en los años setenta con proyectos a distintos niveles para fomentar la redacción clara en el seno del gobierno, como la ordenanza presidencial de 1978 en EE. UU. para hacer la ley "fácil de entender por quienes deben cumplirla" o la formación, en 1994, de la red Plain Language Action and Information Network (PLAIN, https://www.plainlanguage.gov/) de empleados federales estadounidenses que apoyó el proyecto de Ley de Escritura Clara, sancionada en 2010 (Plain Writing Act, 2010). Finalmente, en los años noventa, surgieron diversos proyectos de reforma y reescritura en organismos específicos (Dechter, 2011; James et al., 2015).

En el ámbito profesional, la primera asociación, Clarity International (http://www.clarity-international.org/), dedicada a promover el lenguaje claro en el ámbito jurídico, se fundó en 1983 en el Reino Unido y hoy convoca a profesionales de todo el mundo, incluso de países hispanohablantes. Le siguió Plain Language Association International (https://plainlanguagenetwork.org), PLAIN, sin relación con la red federal estadounidense, en 1995, lanzada en Canadá, que hoy reúne a 
profesionales de muy diversas disciplinas y procedencia y cuya junta directiva a la fecha de redacción cuenta con dos directoras hispanohablantes. Finalmente, en 2003, se creó el Center for Plain Language (https://centerforplainlanguage.org) en Estados Unidos, a fin de deslindar el trabajo de la red federal. Tras la aprobación de la Ley de Escritura Clara, este centro lanzó dos iniciativas para fomentar el uso del lenguaje claro en los ámbitos público y privado: el boletín de calificaciones de organismos federales para evaluar el cumplimiento de la Ley de Escritura Clara y los premios ClearMark Awards para trabajos publicados en Norteamérica tanto en inglés como en español. En el Reino Unido, la Campaña por el Lenguaje Claro La Marca de Cristal es el sello de aprobación de la claridad de un documento. Actualmente aparece en más de 23.000 documentos diferentes en el Reino Unido y en otros países.

Asimismo, comienzan a aparecer iniciativas educativas institucionales como la Plain Language Academy (PLA, https://www.plainlanguageacademy.com) en 2006 y el programa en lenguaje claro del Departamento de Educación Continua en la Simon Fraser University (SFU, https://www.sfu.ca/continuing-studies/programs/plainlanguage-certificate/why-this-program.html) en 2015, ambos ganadores del premio Cheryl Stephens Innovation Award, otorgado por PLAIN. PLA constituye el primer programa de formación integral en lenguaje claro que incorpora, en 2020, un programa multilingüe con una oferta inicial de cursos en inglés, francés y español.

Más allá del mundo angloparlante, los países del norte de Europa son los que han promovido en mayor medida la aplicación del lenguaje claro en ámbitos gubernamentales. Sobresalen Suecia, Noruega y Finlandia con sus institutos de la lengua (ver https://www.sprakradet.no/, respectivamente), que impulsan proyectos de comunicación en lenguaje claro tendentes a informar al ciudadano, asesorar a entes públicos y privados, formar profesionales, y hacer lobby para una mejor implementación del lenguaje claro.

Una iniciativa europea importante atañe a la alfabetización en salud y ha sido especialmente importante durante la pandemia de COVID-19. Se trata del reglamento europeo que exige la redacción de resúmenes legos de ensayos clínicos (Reglamento 536/2014) a fin de promover la confianza, la colaboración y el compromiso de participación de pacientes, cuidadores y el público en general.

Además, el movimiento repercute en todo el mundo. Los gobiernos de Australia y Nueva Zelanda, por ejemplo, han publicado pautas oficiales para el uso del lenguaje claro en la comunicación con el ciudadano (véase https://www.stylemanual.gov.au/formatwriting-and-structure/clear-language-and-writing-style https://www.digital.govt.nz/standards-and-guidance/design-and-ux/content-designguidance/writing-style/plain-language/).

\subsection{El lenguaje claro en el mundo hispanohablante}

En los países hispanohablantes, se destaca la formación de Redes de Lenguaje Claro con el propósito de actualizar la comunicación con el público en los ámbitos judicial y administrativo en compromiso con el derecho a entender y como "puerta de entrada a la democracia" (Poblete y Fuenzalida, 2018: 123). 
La primera iniciativa de estas características surge a finales del siglo XX en España con el Manual de estilo del lenguaje administrativo (1990) del Instituto Nacional de Administración Pública, que, si bien no se define como propuesta de lenguaje claro, aboga por una redacción que priorice la comunicación con el lector y abandone el oscurantismo de los textos administrativos tradicionales. A partir de este proyecto surgieron en España iniciativas en los ámbitos público y privado que han llevado, por ejemplo, a la adopción del proyecto TCR de Transparencia, Claridad y Responsabilidad del banco BBVA (Ossandon, 2017) y a la redacción en lenguaje claro del informe no financiero de IKEA en España (IKEA Ibérica S.A., 2019).

En el ámbito profesional, ha surgido un gran interés por la formación en lenguaje claro, lo que impulsó el lanzamiento de los encuentros EnClaro (https://enclaro.es), coordinados por los equipos profesionales de Cálamo\&Cran y Archiletras y dirigidos por la traductora Isabel Espuelas. En mayo de 2017, por otra parte, se celebró el XII Seminario Internacional de Lengua y Periodismo, bajo el lema "Lenguaje claro, reto de la sociedad del siglo XXI", organizado por la Fundación San Millán de la Cogolla y la Fundación del Español Urgente (http://www.fundeu.es/san-millan-2017/). Tras el seminario, se desarrolló una guía de comunicación clara a cargo de Estrella Montolío y Mario Tascón, (s.f.) a fin de promover el diálogo entre gobierno, empresa y ciudadanía. Recientemente, además, se ha dado a conocer el sistema arText (http://sistemaartext.com/), desarrollado por la profesora Iria da Cunha y que se presenta como una herramienta automática de ayuda a la redacción para la escritura de textos especializados y en lenguaje claro.

En Chile, en el seno de la Biblioteca del Congreso Nacional, se impulsó en 2003 una iniciativa para "eliminar las barreras de comprensión y participación basadas en el lenguaje" (Ferreiro, 2012). Esta iniciativa condujo al desarrollo de guías de preguntas frecuentes sobre derecho en lenguaje llano y al lanzamiento del programa "Ley Fácil" (https://www.bcn.cl/leyfacil), que, hasta el día de hoy, traduce leyes a un lenguaje fácil de entender para una audiencia no experta.

En México, en 2006, la Secretaría de la Función Pública impulsó la creación de un Manual de lenguaje claro (2007) y la capacitación de funcionarios públicos para emitir documentos nuevos y actualizar documentos existentes en lenguaje comprensible al público.

En Colombia, el Departamento Nacional de la Función Pública (DNFP) impulsó la elaboración de la Guía de lenguaje ciudadano para la Administración Pública colombiana, publicada en 2011 (DNFP, 2011) y actualizada en 2015 por el Departamento Nacional de Planeación (DNP) en la Guía de lenguaje claro para servidores públicos de Colombia (DNP, 2015). Durante 2019, se fortalece la iniciativa "Estado simple, Colombia ágil" (2019), impulsada por Función Pública, el Ministerio de Comercio, el Departamento Nacional de Planeación y la Secretaría Jurídica de la Presidencia. Esta iniciativa apunta a la simplificación de trámites y la ágil comunicación con el ciudadano y las empresas, acentuada en respuesta a la pandemia a fin de apoyar la reactivación económica (Función Pública, 2021).

En Uruguay, se lanzó en 2011 el Programa de Lenguaje Ciudadano de la Dirección Nacional de Impresiones y Publicaciones Oficiales (DNIPO), encargada de difundir la 
normativa jurídica, para "fomentar el conocimiento de la normativa nacional por medio de la traducción a un lenguaje llano y simple de la misma, en el entendido que solo con información calificada sobre esta los ciudadanos pueden ejercer plenamente sus derechos y honrar sus obligaciones" (DNIPO, 2011).

En Perú, se publicó en 2014 el Manual judicial de lenguaje claro y accesible a los ciudadanos para apoyar la emisión sentencias claras, aunque la iniciativa se vio revocada por razones políticas (Poder Judicial del Perú, 2014).

En Argentina, el Ministerio de Justicia y Derechos Humanos lanzó el programa Derecho Fácil para promover la comprensión y el uso de la ley. En 2017 se celebraron las Primeras Jornadas de Lenguaje Claro en Buenos Aires, que condujeron al establecimiento de la Red de Lenguaje Claro Argentina. Poco después, la Dirección Nacional del Sistema Argentino de Información Jurídica (SAIJ), dependiente del Ministerio de Justicia y Derechos Humanos de la Nación, publicó la Guía SAIJ de lenguaje claro (2019). Otra iniciativa reciente en el ámbito educativo es la diplomatura en Lenguaje Claro de la Universidad del Salvador(https://diplomaturas.usal.edu.ar/), lanzada en agosto de 2021 y coordinada por las correctoras de textos Claudia Teresa Pelossi y Nuria Gómez Belart.

Todos estos proyectos se centran básicamente en el llamado lenguaje ciudadano utilizado en los ámbitos judicial y administrativo, que busca facilitar el acceso a la información que los ciudadanos reciben del Estado a fin de ayudarlos a entender la ley, cumplir con sus obligaciones y poder ejercer y reclamar sus derechos. Según Poblete y Fuenzalida (2018), "[l]a claridad de las sentencias se convierte en un importante punto de partida para ir acercando la justicia al ciudadano y hacer realidad, de esta manera, el derecho a comprender".

Existen, además, diversas iniciativas y legislación regionales que, por razones de espacio, no se mencionan en este trabajo. Sin embargo, cabe mencionar una vertiente que ha tomado impulso en el mundo hispanohablante: la lectura fácil. Esta iniciativa aplica las directrices internacionales de la International Federation of Library Associations and Institutions (Nomura et al., 2012) a fin de adaptar el contenido a distintos grupos de lectores, como grupos etarios (niños, jóvenes, adultos mayores), personas con discapacidad intelectual o hablantes no nativos, particularmente inmigrantes recientes y refugiados en situación de riesgo.

También en español se reconoce la urgencia del lenguaje claro en la comunicación científico-técnica tanto a nivel especializado como a nivel de divulgación para promover la colaboración interdisciplinaria (Zawoznik, 2021), la legislación basada en la evidencia y la educación del ciudadano (Marazzato Sparano, 2021b).

\subsection{El lenguaje claro de cara al futuro}

Las tres asociaciones de lenguaje claro, con miembros de todo el mundo, conformaron en 2007 la Federación Internacional de Lenguaje Claro o IPLF (https://www.iplfederation.org) para promover la puesta en valor de la profesión. En 2010, diversas voces abogaron, en la publicación de Clarity International, por la necesidad de establecer normas (Harris, Kleimann y Mowat, 2010). En 2019, IPLF 
impulsó un proyecto para la creación de una norma ISO (2015) sobre lenguaje claro. El proyecto fue presentado ante ISO por Standards Australia, la principal organización australiana de normalización, y aprobado en el 2019 por el Comité Técnico 37 para su desarrollo (Balmford, 2020). La norma se encuentra en fase de desarrollo bajo el número 24495 en el seno del grupo de trabajo WG11 dentro del TC37.

En un mundo donde cada vez más se necesita información sociocultural, científica y técnica para tomar decisiones que atañen al bienestar personal, comunitario y ambiental, el empleo de un lenguaje claro en la comunicación con audiencias tanto legas como expertas se hace imprescindible.

\section{Principios de lenguaje claro}

Como se mencionó, a la fecha de esta publicación, se encuentra en desarrollo la normativa ISO 24495 sobre lenguaje claro, que aborda principios para la creación y revisión de textos en lenguaje claro. Si bien la autora forma parte del comité técnico 37, en cuyo seno se desarrolla la citada norma, los lineamientos que se exponen aquí emergen de la práctica profesional de la autora, así como del juicio de expertos con los que la autora trabaja, y de las pautas adoptadas por organizaciones profesionales y educativas de las que forma parte, particularmente PLAIN y Plain Language Academies y organizaciones como la Organización Mundial de la Salud (2017).

El lenguaje claro procura ayudar al lector a encontrar, comprender y utilizar información y se basa en la atención al propósito del texto, a la audiencia a la que ese texto se dirige, a la estructura retórica empleada para transmitir el mensaje, a la expresión misma del mensaje y a su presentación o diseño, así como a la evaluación de su eficacia (PLAIN, s. f.). Estos factores dan lugar a los siguientes principios rectores, propuestos por los expertos mencionados y tomados como punto de partida para la norma ISO aún en proceso de elaboración:

1) Pertinencia del contenido para el propósito buscado, que incluye la selección de temas y objetivos, su explicitación en el texto, y la perspectiva y el nivel de profundidad con que esos temas se tratan.

Para tomar este tipo de decisiones, los autores pueden contar no solo con el juicio de expertos (incluso el propio) y revisar la literatura existente sobre las características de los lectores y el contexto de comunicación, sino también con la participación de lectores meta mediante entrevistas, encuestas, muestreos y desarrollo conjunto (codiseño) del texto en desarrollo. Por ejemplo, en un proyecto para el desarrollo de materiales de educación financiera adaptada a las necesidades de las personas con autismo, el equipo de trabajo utilizó el codiseño con especialistas financieros, personas autistas y sus cuidadores (Autism Québec, 2020).

2) Accesibilidad del contenido en cuanto al soporte de publicación, la estructura retórica y el diseño gráfico del texto, incluida la introducción de elementos no textuales, como imágenes, gráficos o videos, que ayuden a captar y mantener la atención del lector, así como a procesar la información textual.

Cabe notar que el uso del término accesibilidad aquí empleado interseca el sentido de accesibilidad digital (dar acceso a materiales y plataformas informáticas a personas 
con discapacidades); sin embargo, los recursos de accesibilidad digital pueden emplearse sobre un texto no redactado en lenguaje claro. Los lineamientos de la accesibilidad digital están a cargo del Consorcio WWW, en el marco de la Iniciativa WCAG sobre Directrices de Accesibilidad para el Contenido Web (Adams et al., 2021), cuya última versión informa diversas pautas nacionales, como las recomendaciones emergentes de la Ley de Rehabilitación en Estados Unidos, en su enmienda de la de la Sección 508 de 1998 (Rehabilitation Act, 1973), la Ley de Servicios de la Sociedad de la Información y de Comercio Electrónico en España (Ley 34/2002, 2002), la Ley de Accesibilidad de la Información en las Páginas Web en Argentina (Ley 26.653, 2010) o la Ley General para la Inclusión de las Personas con Discapacidad en México (2011), entre otras.

3) Inteligibilidad de la redacción, que responde a requisitos de textualidad y adecuación. La textualidad apunta a la creación del texto siguiendo pautas de gramaticalidad, aceptabilidad, cohesión y coherencia para fomentar su comprensión por la mayor cantidad posible de lectores. La adecuación atiende a la adaptación del estilo o registro a la audiencia principal del texto. Se ahondará en este principio en la sección siguiente.

4) Aplicabilidad del texto al uso previsto, que implica evaluar el documento a lo largo de su ciclo de vida, empleando métodos apropiados para cada etapa, como revisión de literatura sobre la audiencia, entrevistas y encuestas, mapas de calor, codiseño, tests A/B, sondeos de rendimiento, etc.

Este principio del lenguaje claro se relaciona directamente con sus beneficios, de los que se da cuenta en diversas fuentes y sobre los que se debe continuar la investigación. El lenguaje claro ahorra tiempo al evitar errores y, por tanto, la necesidad de clarificaciones (Kloberdanz, 2017) y se constituye en ventaja estratégica, inspirando confianza y credibilidad (García, 2020) al facilitar el intercambio entre interlocutores (sobre todo entre individuos e instituciones: ciudadano y gobierno, cliente y empresa, paciente y sistema de salud, etc.). También conduce a beneficios económicos, porque al ahorrar tiempo ayuda a ahorrar dinero, e incluso a ganarlo (como en el conocido caso del contrato de 180 millones de dólares de British Aerospace, hoy BAE Systems; Maher y Wild, 2002), y porque evita pérdidas que surgen de la mala redacción (como en el caso de un fallo de 5 millones de dólares por la falta de una coma y de estructuras paralelas). Asimismo, el lenguaje claro facilita el cumplimiento de normas, como documenta Joseph Kimble en su ya clásico libro Writing for Dollars, Writing to Please: The Case for Plain Language in Business, Government, and Law (Escribir por dinero, escribir para complacer: en defensa del lenguaje claro en los negocios, el gobierno y la ley, 2012), y se evidencia en diversos recientes proyectos de lenguaje ciudadano. Finalmente, el lenguaje claro demuestra ser de vital importancia en temas de salud y bienestar individual, social y medioambiental. Tanto es así que hoy forma parte integral de proyectos de alfabetización en salud (la capacidad de individuos e instituciones de utilizar información sobre la salud para promover el bienestar propio y ajeno, ODPHP, 2021), como la comunicación con participantes y el público en general en los ensayos clínicos (MRCT, s. f., Reglamento 536/2014), que ha sido de vital importancia en la lucha contra la pandemia de COVID-19. 
A pesar de describirse aquí de manera discreta, estos principios no se aplican secuencialmente durante el proceso de redacción, sino que guían todas las decisiones en las fases de planificación, textualización y revisión de los textos. Por razones de espacio, las siguientes secciones se centran en el principio de inteligibilidad, ya que atañe específicamente al ámbito de intervención de traductores y correctores.

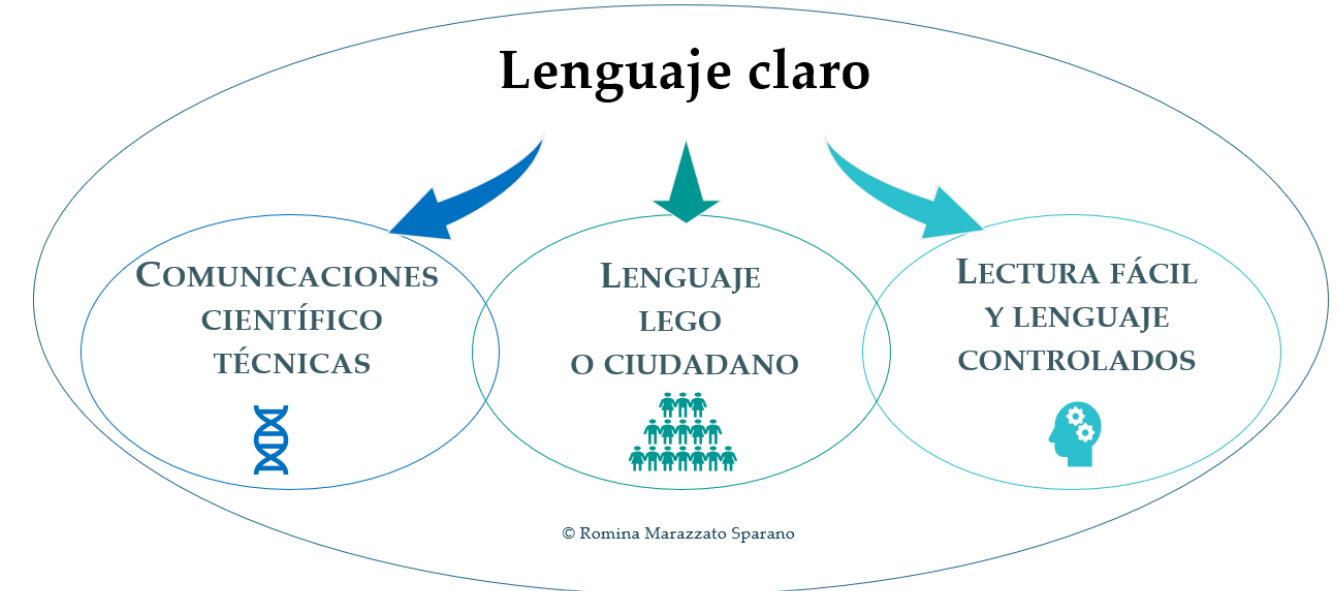

Figura 4. El lenguaje claro puede aplicarse a diversos ámbitos, desde textos especializados a textos legos o de lectura fácil (incluso textos creados en lenguajes controlados)

\subsection{Inteligibilidad}

El principio de inteligibilidad se centra fundamentalmente en la textualidad o textura (Halliday y Hasan, 1988), es decir, en la construcción de significado atendiendo a la gramaticalidad, aceptabilidad, cohesión y coherencia de la redacción, y en la adecuación, es decir, la adaptación de la forma de expresión al lector y al contexto a fin de comunicar con empatía. La empatía juega un rol muy importante en la comunicación eficaz; por ejemplo, en la atención médica, la empatía mejora el diagnóstico al fomentar el intercambio entre profesional y paciente, alienta la toma de decisiones basadas en evidencia y promueve la adherencia al tratamiento (Howick et al., 2020).

Para lograr textualidad, es decir, para construir significado, el autor ha de utilizar el vocabulario, las construcciones y la organización de argumentos que ayuden en mayor medida al lector a comprender el mensaje. A lo largo del texto, el autor debe construir el significado según las reglas gramaticales y las pautas de aceptabilidad dentro de la oración (es decir, las regularidades de un sistema consensuado que constituyen la puerta de entrada a la comprensión), enlazar las oraciones mediante estrategias cohesivas adecuadas y secuenciar las ideas elegidas de forma significativa.

Por ejemplo, para explicar un proceso, se han de presentar sus etapas en una progresión lógica que conduzca al resultado esperado. En la oralidad, en cambio, es posible volver sobre lo dicho y agregar elementos o pasos omitidos, pero, en la escritura, el orden azaroso debe corregirse para evitar confusión y errores. 
Sin duda, la textualidad se ve afectada por la selección de contenido según las necesidades y conocimientos previos del lector (pertinencia), la superestructura de un género textual, los patrones organizativos empleados y su presentación visual (accesibilidad), las elecciones léxicas y estilísticas que corresponden al registro (adecuación) y la atención a los elementos de evaluación disponibles para asegurar la eficacia del texto (aplicabilidad). Ahora bien, todos estos principios se pueden respetar en un texto que, sin embargo, viola el principio de textualidad.

La adecuación, como requisito para la inteligibilidad, tiene que ver con el posicionamiento del texto en su entorno cultural, sobre todo como parte de un género socialmente consensuado. El autor debe adaptar el contenido no solo en cuanto a la selección de temas y a la profundidad con que se han de tratar, sino también en cuanto al registro de la redacción para apoyar la comunicación con el lector previsto. Nótese que entendemos por registro el aspecto interpersonal del texto según lo define Eggins (2011: 84-87), más allá de la convencional distinción entre registros formal e informal.

Un aspecto importante de adecuación, en el que no se ahondará por razones de espacio, se relaciona con la comunicación de empatía mediante el uso de un lenguaje libre de sesgo. Este concepto se ha formalizado en las pautas de estilo sobre respeto y objetividad de la American Psychological Association o APA (2020: 131-149), fundamentalmente dirigidas a la investigación, sobre todo en el caso de descriptores de individuos y grupos que son sujetos de investigación, pero útiles en otros ámbitos. Un lenguaje libre de sesgo requiere de la toma de conciencia sobre estereotipos, prejuicios y omisiones, y del cuestionamiento sobre la pertinencia, precisión y especificidad de los términos que se han de emplear. Dos nociones importantes a estos efectos son la idea de interseccionalidad, es decir, el reconocimiento de que las personas pertenecen a la vez a diversas categorías, y la perspectiva de identificación, es decir, el punto de vista desde el que se identifica el grupo o individuo. Por ejemplo, en el mundo de la discapacidad, puede hacerse hincapié en la persona ("una niña con epilepsia" en lugar de "una niña epiléptica") o en la discapacidad ("los ciegos" en lugar de "las personas con ceguera"). Al considerar qué aspectos de una identidad multifacética corresponde expresar, la APA recomienda tener en cuenta la autodenominación (cómo se autodescribe el grupo o individuo en cuestión) y optar por terminología positiva ("usuario de silla de ruedas" en lugar de "*confinado a una silla de ruedas").

Los ejemplos de adecuación suelen incluir la adaptación de información técnica a un público lego en la materia, que es, de hecho, una posibilidad válida y frecuente, como en el caso de los comunicados de prensa o la cobertura periodística de descubrimientos científicos. También la adecuación técnica es necesaria para la comunicación entre pares e incluye, por ejemplo, el respeto de las normas de estilo o la superestructura esperada en un determinado género textual. Este aspecto de la adecuación técnica incluye el uso escrupuloso de términos técnicos, que "se elaboran diligentemente para reflejar capas de significado muy preciso para un experto" (Sue Ellen Wright, comunicación personal, 9-12-2019).

Pueden considerarse también aquí las adaptaciones de la llamada lectura fácil, cuyo objetivo es brindar acceso a información escrita a personas con discapacidades intelectuales o en situación de riesgo o exclusión social (Pérez García y López de la Cruz, 
2015). Otro tipo de adaptaciones incluyen el uso de lenguajes controlados, que establecen un repertorio léxico y gramatical explícitamente restringido a fin de promover la legibilidad y evitar la ambigüedad de textos en disciplinas específicas. Un ejemplo de este tipo de lenguajes para humanos (también existen lenguajes controlados orientados a máquinas) es el inglés técnico simple (ASD, 2017), desarrollado para ayudar a los lectores de documentación aeroespacial. El uso de lenguajes controlados es especialmente relevante en el ámbito de la traducción (Mitamura y Nyberg, 2001; Ramírez Polo, 2014).

\section{El lenguaje claro en acción: técnicas de expresión para mejorar la inteligibilidad}

Como se mencionó, en este trabajo se hace hincapié en la aplicación del principio de inteligibilidad, que atañe específicamente a las etapas del proceso de redacción en las que se centran las intervenciones del traductor y el corrector. Estos profesionales no suelen tener injerencia directa en la selección de contenido (pertinencia), la determinación del tipo textual y soporte mediático (accesibilidad) o la intención y evaluación de la efectividad del texto (aplicabilidad).

Esto no significa que no existan instancias relativas a estos otros principios en las que traductores y correctores no puedan intervenir. Así ocurre en el ejemplo siguiente sobre acción climática, donde la mención del año 2040 no se dirime en el resto del texto. Será responsabilidad del corrector o del traductor hacer notar esta falencia para encontrar una solución.

1. a. ?L'utilisation du charbon pour la production d'électricité doit diminuer, au niveau mondial, de $80 \%$ d'ici à 2030, par rapport aux niveaux de 2010. Cela signifie que les économies développées doivent s'engager à avoir éliminé le charbon d'ici à 2030, et les autres pays, d'ici à 2040.

b. ?El consumo mundial de carbón para generar electricidad debe reducirse en 2030 en un $80 \%$ por debajo de los niveles de 2010. Eso significa que las economías desarrolladas deben comprometerse a eliminar el consumo para 2030 y que los demás países deben hacerlo para 2040.

Para organizar las intervenciones en lenguaje claro, se propone la conceptualización de un sistema bidimensional cuyos ejes representan la progresión de la textualidad, de textos oscuros a textos claros, y de la adecuación, de textos especializados a textos legos. Por supuesto, la dirección de las revisiones (a lo largo del eje de la textualidad, a lo largo del eje de la adecuación o de ambos) dependerá, según explicitan normas como las ASTM e ISO ya citadas, de las especificaciones de cada proyecto.

En la figura 1, se ofrece un ejemplo de aplicación de este sistema. Se trata de un breve fragmento destinado a cuidadores de pacientes titulado "Diagnóstico y tratamiento de la policitemia vera". La policitemia vera es un tipo de cáncer de la médula ósea. A partir de un texto técnico poco claro en el cuadrante inferior izquierdo, se puede 
apreciar una revisión a lo largo del eje de la adecuación, que resulta en un texto no técnico, pero igualmente oscuro, en el cuadrante inferior derecho. En cambio, si se revisa primero el texto a lo largo del eje de la claridad, se obtiene un texto técnico claro en el cuadrante superior izquierdo, a partir del cual se puede luego generar un texto lego claro en el cuadrante superior derecho.

\begin{abstract}
En un $20 \%$ de los casos, la sobreproducción de eritrocitos deriva en fibrosis medular. La progresión fibrótica desencadena hematopoyesis extramedular en el bazo, lo que, a su vez, resulta en esplenomegalia.
\end{abstract}

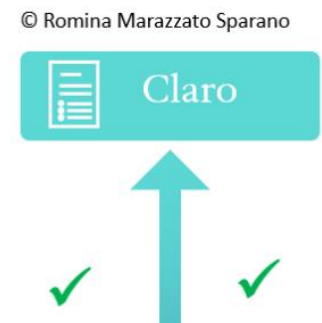

En un $20 \%$ de los casos, la médula ósea se ve invadida por tejido fibroso cicatrizante. Con el tiempo, la médula no puede producir suficientes glóbulos rojos. El cuerpo compensa esta falta produciendo glóbulos en el bazo. Esto hace que el bazo se agrande de manera anormal.

\section{Técnico}

?Con la progresión de la enfermedad se produce esplenomegalia en un $20 \%$ de los casos pues la médula ósea muestra crecientes signos de fibrosis, lo que causó hematopoyesis extramedular durante el curso de la enfermedad.

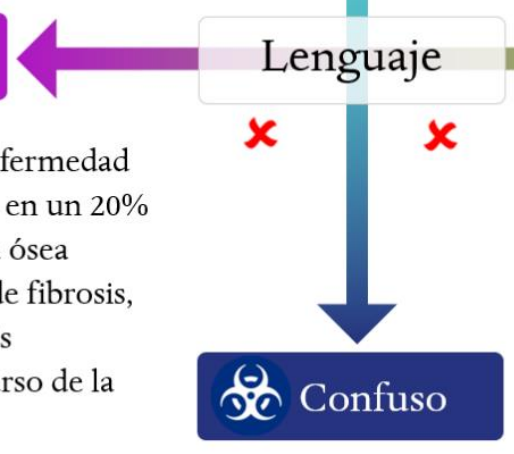

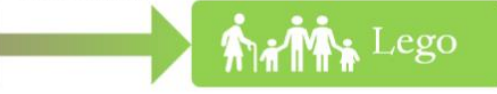

?A medida que la enfermedad progresa, aumenta el tamaño del bazo en un $20 \%$ de los casos. Esto se debe a que aparece cada vez más tejido cicatrizante (fibrosis) en la médula ósea. Entonces el cuerpo produce glóbulos sanguíneos fuera de la médula a medida que la enfermedad avanza.

Figura 5. Ejemplo de revisión iterativa empleando un sistema bidimensional para abordar la inteligibilidad, con la textualidad representada en el eje vertical y la adecuación, en el eje horizontal

Seguidamente, se exploran intervenciones sobre todo en el eje de la textualidad, dado que este eje, como se aprecia en el ejemplo anterior, es esencial para la claridad. Por otro lado, la adecuación (el cambio de registro, el ajuste terminológico, etc.) depende en mayor medida de las especificaciones de cada proyecto.

Se abordan primero problemas de redacción que surgen de fallas en la construcción de significado y deben resolverse para que el texto pueda ser comprendido en tanto texto monolingüe de partida (para su posterior traducción, por ejemplo) o texto traducido de llegada. Se abordan luego algunos problemas de traducción que surgen de la falta de correspondencia entre las posibilidades de expresión de cada idioma y deben resolverse para evitar el sinsentido o la oscuridad de un texto altamente extranjerizado en un contexto en el que esta estrategia no tiene cabida (como es el caso de textos informativos sobre salud, medidas impositivas, beneficios sociales, etc.).

\subsection{Cuestiones de redacción}

Los problemas de redacción atañen a la gramaticalidad, aceptabilidad, cohesión y coherencia de un texto. En la redacción de un texto original (en tanto que va a ser traducido o corregido), los problemas se presentan y resuelven en las diferentes etapas 
del proceso (planificación, textualización y revisión). Para abordar estos problemas en la traducción y la corrección, es necesario tanto el bagaje profesional como el diálogo con el autor del texto original, dado que el objetivo de un texto informativo es aportar conocimiento al lector (ya sea enciclopédico, conceptual, procedimental o metacognitivo; Krathwohl, 2002). Particularmente en la traducción, aun cuando no se tenga injerencia sobre el texto de partida ya publicado, a menudo es posible desambiguar o corregir el texto de llegada para aumentar su comprensibilidad.

\subsubsection{Gramática y aceptabilidad}

La gramaticalidad ha sido el factor más explorado entre los requisitos de una buena redacción, pues se refiere al cumplimiento de normas (convenciones, por lo general explícitas, que surgen de "regularidades estadísticas codificadas en la conectividad de la red neuronal", Nadau, 2012: 63) en la construcción de palabras, frases y oraciones. Entre las pautas de gramaticalidad se cuentan la concordancia entre sujeto y verbo, la puntuación y el uso adecuado de pronombres en las cadenas de referencia. En el siguiente ejemplo, se ilustra cómo se puede esclarecer el texto de llegada corrigiendo una falta de referente. El texto aparece en un folleto para familiares de personas que sufren de alcoholismo:

2. a. Alcoholism is a devastating disease, destroying not only their own life, but that of loved ones around them.

b. *El alcoholismo es una enfermedad devastadora, que destruye no solo su propia vida, sino la de los seres queridos que los rodean.

c. El alcoholismo es una enfermedad devastadora, que no solo destruye la vida de la persona afectada, sino la de sus seres queridos.

En 2.b, "su propia vida" no tiene referente o se lee deícticamente como dirigido al lector cuando la intención no es esa; por eso, debe explicitarse el antecedente de "su".

La aceptabilidad incorpora un componente pragmático por el cual las frases y oraciones no solo deben responder a las reglas gramaticales, sino también a pautas del sentido común que las hagan aceptables para los lectores (que, eventualmente, pueden incluso redefinir la gramaticalidad; Leivada y Westergaard, 2020). Entre las estrategias de aceptabilidad figuran, por ejemplo, el uso de estructuras paralelas que ayudan a procesar elementos del mismo nivel conceptual y la especificación de referencias (pasar de una frase nominal indefinida a una definida, luego a un nombre propio y, finalmente, a un pronombre). En el ejemplo siguiente, correspondiente a un texto de divulgación sobre la técnica de andamiaje en educación, el traductor o corrector puede reparar la falta de una estructura paralela:

3. a. ?Scaffolding is breaking up the learning into chunks and then you provide a tool, or structure, with each chunk.

b. ?El andamiaje consiste en dividir el aprendizaje en segmentos y luego se proporciona una herramienta o estructura para cada uno de ellos.

El andamiaje consiste en dividir el aprendizaje en segmentos y luego proporcionar una herramienta o estructura para cada uno de ellos. 


\subsubsection{Cohesión y coherencia}

La cohesión y la coherencia del texto tienen que ver con "la continuidad que existe entre una parte del texto y otra" (Halliday y Hasan 1976: 299) y la adecuación al tema y objetivo del texto (Giora, 1985). La cohesión es el aglutinante visible del texto que aparece a nivel léxico y gramatical para expresar relaciones referenciales, lógicas, estructurales y pragmáticas dentro del texto, mientras que la coherencia es un aspecto menos tangible que emerge de la unidad temática general y la existencia de relaciones, señaladas o no, que responden al mantenimiento de la pertinencia (o relevancia) a lo largo del texto (Portolés, 1994).

Las estrategias presentadas aquí apuntan a características inherentes al texto que ayudan al lector a construir el significado previsto, aunque cabe notar que la recreación de la coherencia por parte del lector nunca logra una réplica exacta de la planeada por el autor (Bulitz et al., 1999).

Entre las estrategias útiles para ajustar la cohesión y la coherencia textual se cuentan:

- La reiteración de conceptos, mediante la repetición literal, el uso de sinonimia, hiperonimia e hiponimia, o la definición. Por ejemplo, las frases nominales Jacinda Ardern, la primera ministra o la mandataria de turno pueden referir a la misma persona en el contexto del gobierno de Nueva Zelanda en 2021.

- El uso de marcadores para señalar expresamente relaciones lógicas, estructurales o pragmáticas en el texto: como, sin embargo, mientras, por un lado, en resumen, irónicamente, además, quizás, etc. Sin duda, no es siempre posible para el traductor o el corrector reponerlos en el texto, pero se trata, sin embargo, de una herramienta que no se puede desconocer.

- La redistribución sintáctica, para reubicar frases dentro de la oración, a fin de cambiar la perspectiva o el énfasis de la información presentada y así resaltar relaciones entre diversos participantes o circunstancias del evento o reordenar frases, ya sea para empezar por información conocida (y facilitar la introducción de información nueva) o para empezar por información nueva (y llamar la atención del lector).

- Las cadenas de referencias que requieren del claro posicionamiento de antecedentes y referentes.

En el siguiente ejemplo, procedente de un informe de investigación sobre la enseñanza de la lectoescritura, la oración es correcta gramaticalmente e, incluso, adecuada a su público. Sin embargo, cuando se la considera atentamente, surge un quiebre debido a una referencia errónea que el corrector o el traductor (en este caso del español al inglés) deben resolver para agilizar la lectura.

4. a. ?Son pocas las maestras que a la edad de cinco años pueden enseñarles a los niños a leer.

b. ?Few are the teachers who, at the age of five, can teach children to read.

c. Son pocas las maestras que pueden enseñarles a los niños a leer a la edad de cinco años.

d. Few are the teachers who can teach children to read at the age of five. 
La frase preposicional a la edad de cinco años no se refiere a la edad de las maestras, el sustantivo previo, sino a la edad de los niños, un sustantivo presentado con posterioridad a la frase. Para evitar la ambigüedad, se puede desplazar la frase hacia el final de la oración a fin de acercarla al antecedente previsto, en lugar de contar con que el lector la vaya a dirimir intuitivamente.

Quizás un elemento puente entre cohesión y coherencia sea la manipulación del flujo informativo, que depende, básicamente, de cómo se presentan el tema y el rema en cada oración y cómo se concatenan a lo largo del texto - de manera lineal, constante, derivada, etc. (Daneš, 1974; Eggins, 2001). En lenguaje claro, la manipulación del flujo informativo cumple una función fundamental en la construcción de la textualidad. En particular, la redistribución sintáctica dentro de la oración permite manipular el flujo informativo sin alterar los roles semánticos de los participantes del evento referido (Pinker, 2014: 35) y se transforma en una estrategia esencial de cohesión (Marazzato, 2021a). Entre otras técnicas de redistribución se cuentan:

- El desplazamiento de frases; particularmente, la frontalización se usa a menudo para situar eventos en tiempo y lugar literal o metafóricamente. Por ejemplo, la famosa apertura del Quijote, "En un lugar de la Mancha", frontaliza un complemento de lugar.

- La voz pasiva, que permite presentar un evento transitivo con el objeto como tema o punto de partida cuando el agente es desconocido o irrelevante, se erige en noticia respecto del objeto y merece la posición remática, o simplemente debe explicitarse en una larga frase que impone una gran carga cognitiva para el lector. Por ejemplo, en el resumen de una de las versiones del mito de Edipo, la voz pasiva echa luz sobre Edipo niño a la vez que permite explicar un aspecto complejo del agente del evento: "El bebé fue rescatado por un pastor, cuyo mandato había sido abandonar al niño". Nótese que no funcionaría la oración en voz activa.

- La copulativa enfática u oración escindida, que incluye diversos tipos de fórmula de relieve para focalizar un elemento oracional desplazándolo de su ubicación canónica. Por ejemplo, la oración enfática de relativo, como en "Era ella quien le chupaba la sangre y no yo", o la enfática con que galicado como "Fue allí que la imputada subrepticiamente tomó de pasada la cuchilla y la ocultó" (ambas citas: Puig, Boquitas pintadas).

- El uso de pares verbales como dar/recibir o comprar/vender, que permiten cambiar el punto de observación del evento.

La redistribución permite ajustar la perspectiva desde la que se presenta el evento, ayuda al lector a procesar la relación entre referentes y antecedentes y aligera el procesamiento del texto dinamizando el flujo informativo. En los ejemplos siguientes, la redistribución sintáctica permite manipular el impacto que los diversos participantes del evento tienen en la oración. En 5.c, por ejemplo, al desplazar al inicio de la oración el adjunto de tiempo, se esclarece que debe leerse un significado iterativo respecto de la aparición del dolor y no durativo respecto de la duración de la posición. En 6.c, el uso de la voz pasiva evita la repetición de un actor conocido del evento (los investigadores), a la vez que se resalta su agentividad en el desarrollo del cuestionario empleado al presentarlo en el rema oracional.

5. a. The patient complains of chest pains if she lies on her left side for over a year. 
b. *La paciente refiere dolores de pecho si se recuesta sobre el lado izquierdo durante más de un año.

c. Durante más de un año, la paciente refiere dolores de pecho cuando se recuesta sobre el lado izquierdo.

6. a. For the collection of sociodemographic data, the researchers used a questionnaire that they designed themselves.

b. Para la recolección de los datos sociodemográficos, los investigadores utilizaron un cuestionario que diseñaron ellos mismos.

c. Para la recolección de los datos sociodemográficos, se utilizó un cuestionario diseñado por los investigadores.

A continuación, se presenta un ejemplo de un texto algo más extenso, que requiere atender a aspectos organizativos de coherencia, en el resumen de un artículo médico titulado "Reevaluación de la histerectomía". Para intervenir, sin duda, se requiere de una abierta comunicación con el autor original.

7. a. ORIGINAL INGLÉS

The surgery performed to remove the uterus is called a hysterectomy. The removal of the uterus relieves symptoms caused by medical conditions affecting this organ. It is usually only considered if other treatments aren't effective enough. It is a major surgical procedure that is associated with risks and side effects. Hysterectomy may be necessary to remove the tumor if a woman has uterine or ovarian cancer.

b. PRIMERA VERSIÓN EN ESPAÑOL

La intervención quirúrgica para extirpar el útero se denomina histerectomía. La extirpación del útero alivia síntomas causados por enfermedades que afectan a este órgano. Por lo general, sólo se considera si otros tratamientos no son lo suficientemente eficaces. Se trata de una intervención quirúrgica importante que conlleva riesgos y efectos secundarios. La histerectomía puede ser necesaria para extirpar el tumor si una mujer tiene cáncer de útero o de ovarios.

c. VERSIÓN REVISADA EN ESPAÑOL

La histerectomía es una operación para extirpar el útero. La extirpación del útero alivia los síntomas causados por enfermedades que afectan a este órgano. Si una mujer padece de cáncer de útero o de ovarios, por ejemplo, la histerectomía puede ser necesaria para extirpar el tumor. Sin embargo, se trata de una intervención quirúrgica importante que conlleva riesgos y efectos secundarios. Debido a estos riesgos, normalmente solo se la considera si otros tratamientos no son lo suficientemente eficaces.

En 7.b, al analizar la función de las cinco oraciones que constituyen el texto y su flujo informativo, aparece una organización de tipo lluvia de ideas, sin orden, que no promueve su procesamiento por parte del lector. Se presentan las restricciones al uso de la cirugía y sus riesgos en el centro del texto. Al cierre del texto, que ejemplifica una instancia en la que se hace necesaria la intervención, se genera una contracción con el propósito explícito de reevaluar su empleo - pasar de única o mejor opción de tratamiento a ser un recurso de última instancia. 


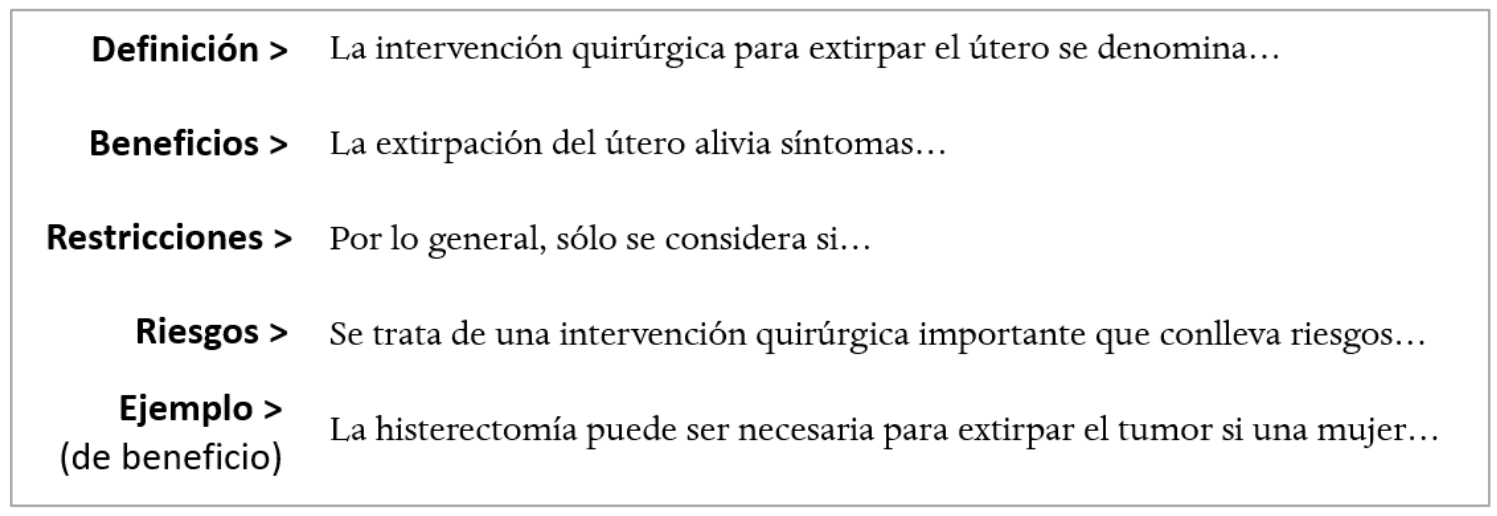

Figura 6. Función de cada oración en la versión original

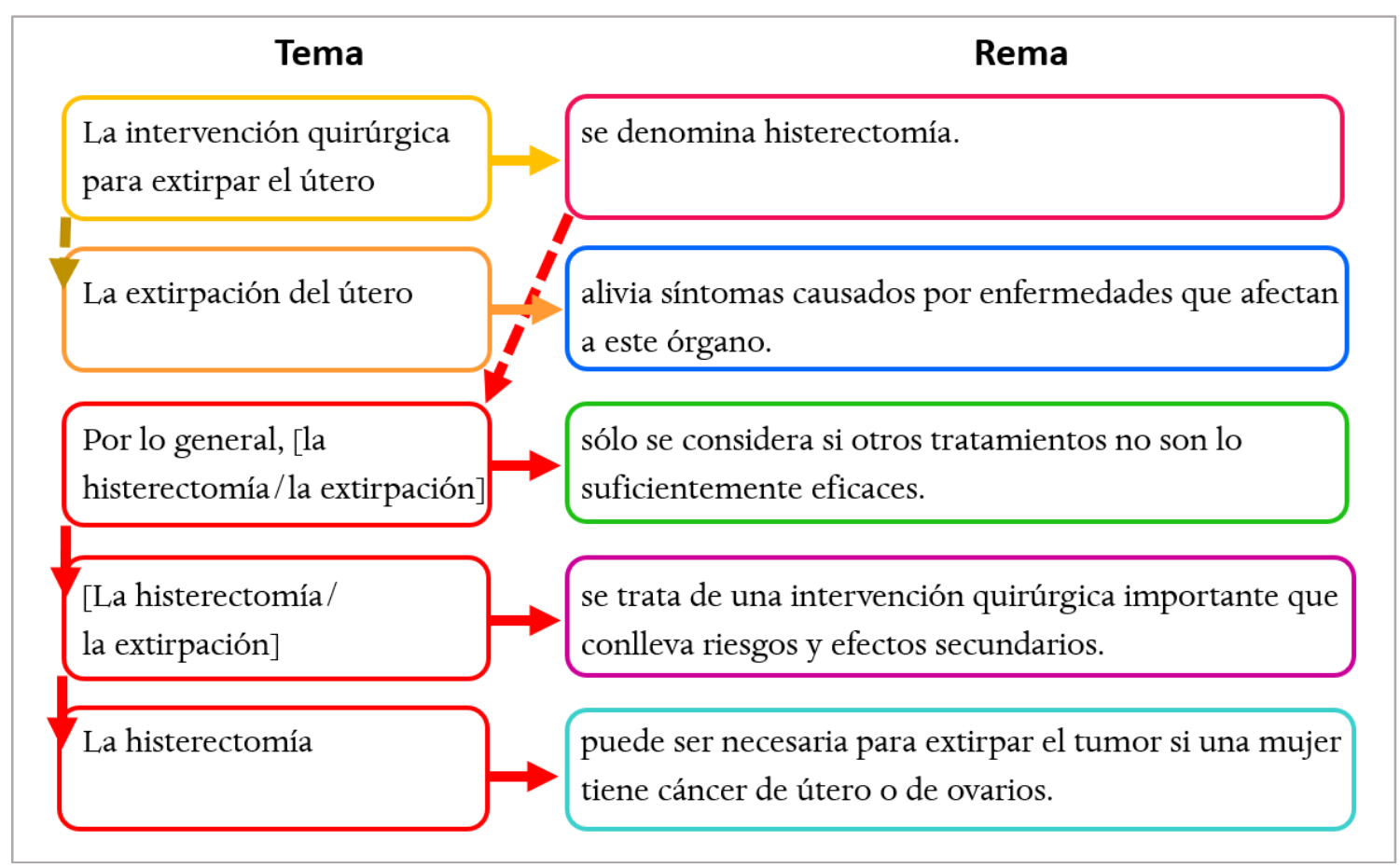

Figura 7. Visualización del flujo informativo en la versión original

Una posible reorganización de las ideas debe responder mejor a la expectativa presentada en el título de reevaluar el procedimiento. Cabe recordar que el título de un texto activa, como demostraron Bransford y Johnson (1972), el conocimiento previo del lector y ayuda a predecir y recordar su contenido. Así pues, un orden que atienda tanto a la progresión de la información como a la función de cada oración en un patrón argumental al estilo rogeriano (Brent, 1996) ha de contextualizar el procedimiento para luego concluir en la reevaluación. Por ejemplo, puede presentar una explicación en lugar de una definición en la primera oración, seguidamente el reconocimiento de los beneficios de la cirugía, luego un ejemplo de cuándo es necesaria y, finalmente, los riesgos que comporta (como explicitación del contexto), para concluir con las restricciones que estos riesgos imponen a su uso, es decir, la anticipada reevaluación. 
Explicación > La histerectomía es una operación para extirpar el útero.

Beneficios > La extirpación del útero alivia los síntomas causados por...

Ejemplo > Si una mujer padece de cáncer de útero o de ovarios, por ejemplo...

Riesgos > Pero se trata de una intervención quirúrgica importante que conlleva riesgos...

Restricciones > Debido a estos riesgos, normalmente sólo se la considera...

Figura 8. Función de cada oración en el texto revisado

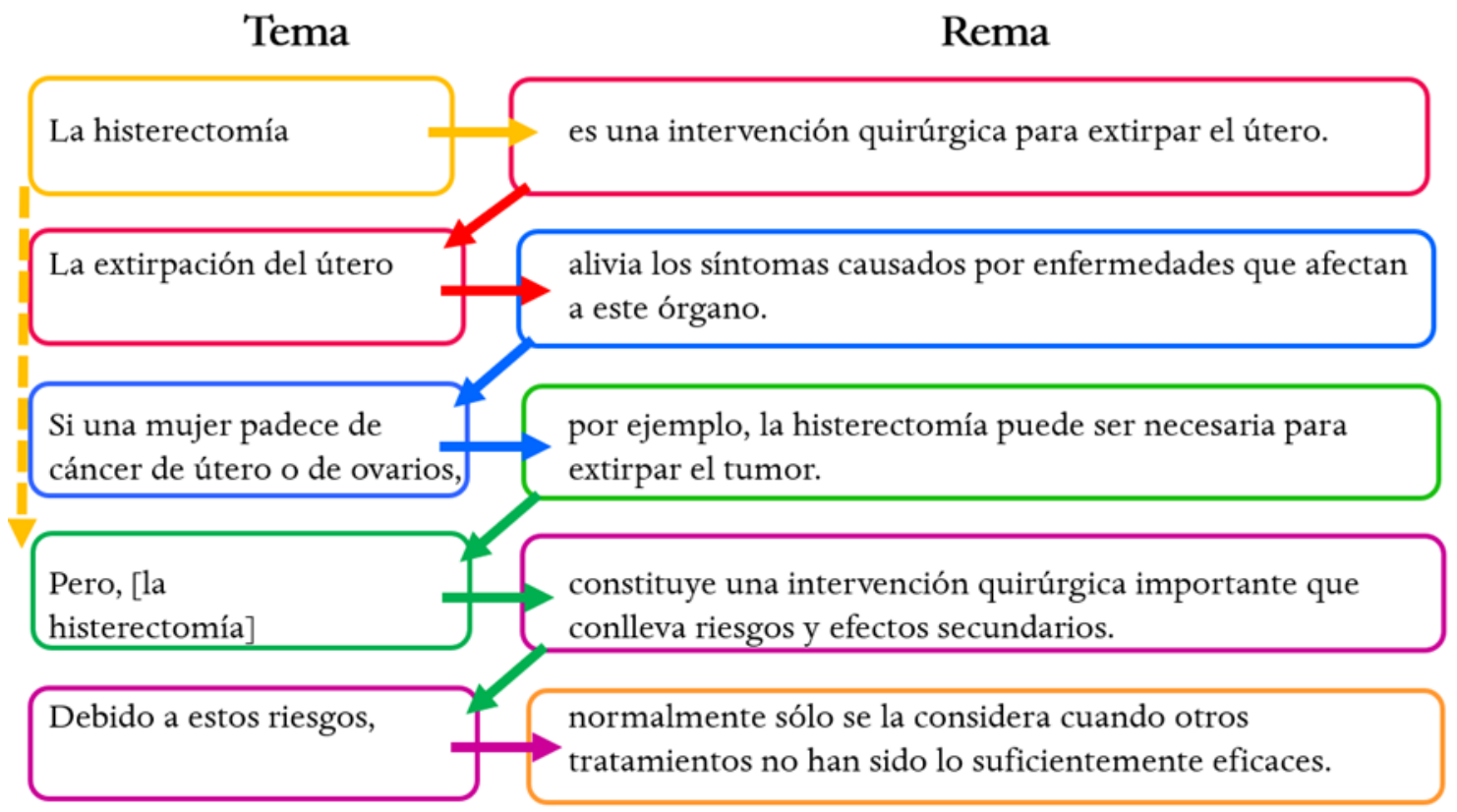

Figura 9. Visualización del flujo informativo de la revisión en lenguaje claro

\subsection{Posibilidades de expresión}

Las posibilidades de expresión se refieren a las características idiosincráticas de cada lengua que permiten la construcción, representación y expresión de la realidad de modos diversos y acarrea problemas de traducibilidad.

La traducibilidad se ha abordado desde diversas perspectivas, que van desde el llamado a la extranjerización de Venuti (2004) para marcar divergencias ideológicas (respecto de la norma anglófona y en textos sobre todo literarios) o al distanciamiento de Sturge para reconocer la otredad hasta el resurgimiento de la transcreación como estrategia de domesticación extrema (Munday, 2016), en el sentido establecido por Venuti, a partir de la reinterpretación del original y la creación de nuevas construcciones conceptuales, lingüísticas y culturales para suplir su falta o la insuficiencia de las existentes (Gaballo, 2012). (Para un breve resumen sobre el complejo tema de equivalencia, en cuanto al léxico, la sintaxis, el texto en su totalidad o sus implicaciones pragmáticas, ver Panou, 2013). Se ofrecen aquí unas breves observaciones sobre el uso de principios de lenguaje claro para resolver dificultades concretas.

En el continuo de opciones en la traducción, el lenguaje claro, por aplicarse fundamentalmente a textos informativos, apela al uso de estrategias de domesticación 
a fin de garantizar su "inteligibilidad inmediata". Es difícil promover la comprensión tendente a la toma de decisiones o la ejecución de tareas cuando se fuerzan las posibilidades de expresión de una lengua. El mismo Venuti reconoce las exigencias comunicativas en los textos informativos, que se intercambian entre naciones, instituciones e individuos para apoyar la actividad económica, el progreso científicotécnico, el intercambio sociopolítico y la salud.

En el ejemplo siguiente, extraído del folleto mencionado sobre alcoholismo, el texto en inglés emplea palabras y estructuras ajenas al español que el traductor debe adaptar.

8. a. When a loved one is drinking their life away, you can feel disempowered and lost.

b. *Cuando un ser querido se está bebiendo su vida/bebe demasiado, puedes sentirte desempoderado y perdido.

c. Cuando un ser querido malgasta su vida en el alcohol, puedes sentirte desvalido y sin rumbo.

Se analizan en las siguientes secciones las discrepancias léxicas y las discrepancias sintácticas.

\subsubsection{Discrepancias léxicas}

Todas las lenguas nombran sus realidades, unas comunes a cualquier experiencia humana y otras solo relevantes en su cultura. Las diferencias emergen al captar realidades diversas, rescatar distintos aspectos de la percepción del mundo, expresar la experiencia psicológica de esas percepciones (en urdu, por ejemplo, la palabra naz designa orgullo que uno siente al saber que es amado, un sentimiento probablemente existente pero no lexicalizado en todos los idiomas) y las creencias que se construyen a partir de percepciones y creencias (la palabra china yuanfuen significa algo así como "la fuerza que nos conecta a todos", un concepto profundamente ligado a la práctica budista). A estas diferencias se agregan discrepancias en la carga connotativa y en los valores metafóricos de los ítems léxicos en el sentido presentado por Lakoff y Johnson (2003), relacionados con el concepto de equivalencia funcional (o dinámica) ya presentado por Nida (1969).

En el ejemplo 8, la dificultad léxica surge en la palabra disemplowered que remite el concepto de empowerment. Si bien en español se ha acuñado la palabra empoderamiento, se trata de un vocablo, y de un concepto, mucho menos extendido y de aplicación más restringida que la que tiene el término en inglés (Calvès, 2009). Tratándose de un texto tendente a apoyar a familiares en una situación angustiante, se debe buscar un término que genere mayor empatía con el lector, como "desvalido".

\subsubsection{Discrepancias sintácticas}

Las discrepancias sintácticas surgen de constructos que codifican la realidad de distintas maneras. Quizás una de las diferencias idiosincráticas más profundas entre el inglés y el español sea la diferencia de marco (Talmy, 1972, 2000; Slobin, 2004). La noción de marco se aplica a la conceptualización de eventos en los que un evento 
principal enmarca a otro en una relación de fondo y figura en la que cada lengua (es decir, cada cultura) establece qué es fondo y qué es figura.

El inglés es una lengua de marco satelital que expresa el resultado de un evento mediante satélites o adjuntos al verbo, generalmente preposiciones (go out, come in, step away). En cambio, el español es una lengua de marco verbal que expresa el resultado en el verbo principal (salir, entrar, ausentarse). Existen lenguas, llamadas de marco equipolente, que presentan ambos mecanismos. Cabe notar que el inglés utiliza una serie de construcciones de marco verbal heredadas del francés, que pertenecen generalmente a un registro formal (descend frente a go down, postpone frente a put off). Un indicador de la diferencia entre lenguas de marco satelital y verbal es la cantidad de preposiciones de cada idioma: el inglés supera ampliamente al español en este aspecto (Marazzato, 2018). Esta diferencia también ha sido explorada desde la perspectiva de las neurociencias, que encuentran diferentes patrones en la recuperación funcional de lesiones cerebrales de zonas que albergan la representación de estos diversos aspectos del verbo en distintos idiomas (Nadau 2012: 99-105).

La diferencia de marco genera una aparente inversión en el orden de las palabras, ya observada por Vinay y Darbelnet (1977: 105). Sin embargo, se trata de algo más que de un entrecruzamiento (chassé-croisé), como lo denominaron estos autores. La expresión de resultado en un satélite libera al verbo enmarcado de carga semántica, y esto le permite incorporar léxicamente otros aspectos del evento, como la manera, como se observa al traducir run out por salir (corriendo), donde la manera en español puede obviarse sin pérdida de gramaticalidad.

Este proceso de incorporación de la manera al núcleo verbal se denomina conflación de manera (Talmy, 1972: 242) y posibilita una mayor carga semántica en los verbos compuestos del inglés. En español, en tanto lengua de marco verbal, se suele obviar la manera, por presuponerse, de modo que es innecesario aclarar el resultado en un satélite por estar ya lexicalizado en el verbo; por eso no resultan aceptables las frases *salir afuera o *entrar adentro.

Al traducir este tipo de verbos con conflación de manera, las lenguas romances tienden a perder la expresión de manera (Slobin, 2000). El lenguaje claro apoya esta tendencia idiosincrática, a menos, por supuesto, que la manera tenga una peculiar relevancia. Por ejemplo, normalmente "a bird flew into the barn" se traducirá como "un pájaro entró al establo"; en cambio, "a bird limped into the barn" reclama la aclaración de manera, "un pájaro entró rengueando al establo".

Asimismo, la conflación de manera permite la construcción de frases resultativas como "wipe the surface clean", "storm into the room" y "drink your life away". En español, este tipo de construcciones es muy poco frecuente y tiende a usarse junto a expresiones que enfatizan el resultado, como el adverbio bien en el aviso sobre el insecticida Raid:

9. a. [Raid] kills them dead.

b. [Raid] las mata bien muertas.

Así pues, en la traducción al español, se ha de preferir expresar el resultado de la construcción. En el ejemplo siguiente, de un informe sobre la falta de adherencia a un procedimiento de desinfección, es importante desambiguar el motivo de la falta de efectividad del producto: 
10. a. He immediately wiped the table dry, without giving the product time to act.

b. ?Le pasó inmediatamente un trapo a la mesa para secarla, sin dar tiempo a que el producto actuara.

c. Secó inmediatamente la mesa (con un trapo) sin dejar actuar al producto.

En 10.b, se resalta la manera (pasar un trapo) y la intención (para) de un resultado (secar) al que no necesariamente se llega, mientras que en 10.c se expresa el resultado, pudiéndose obviar la manera.

Se señala también que otras estructuras idiosincráticas de interés a la hora de traducir y corregir teniendo en cuenta las pautas del lenguaje claro son la nominalización y el sistema de clíticos dativos, aunque no se exploran aquí por razones de espacio.

\section{Conclusión}

En este artículo se presenta un panorama del lenguaje claro en tanto enfoque para la redacción y en tanto movimiento alineado con el derecho a entender como derecho civil, y se explora su rol en textos informativos (de contenido científico-técnico, legal o administrativo), con particular interés en su aplicación a la traducción y la corrección.

En la sección 1, se define el lenguaje claro como el uso de redacción, estructura y diseño que permiten al lector meta encontrar lo que necesita, comprender lo que encuentra y usar esa información. En la consideración de la claridad del texto, se toman nociones de lingüística funcional para definir el texto y presentar técnicas de expresión que promuevan la claridad. Se entiende que el lenguaje claro se aplica primordialmente a textos informativos que apuntan a que el lector comprenda o aprenda nuevos conceptos, adquiera nuevas habilidades, tome decisiones informadas o realice una tarea o proceso. El tipo textual informativo se entiende desde una perspectiva funcional a partir de su función referencial y puede actualizarse en diversos géneros. También se apuntan algunas cuestiones sobre el uso de fórmulas de legibilidad.

En la sección 2, se repasan algunos hitos del movimiento de lenguaje claro, tanto en los países angloparlantes como en el mundo hispanohablante, que llevan a la formación de la Federación Internacional de Lenguaje Claro (IPLF), integrada por tres asociaciones de lenguaje claro. Se señala que IPLF ha impulsado la elaboración de la norma ISO 24495 Lenguaje claro: principios rectores y lineamientos, actualmente en desarrollo.

En la sección 3, se exponen brevemente los principios del lenguaje claro partiendo de las propuestas de los expertos de las asociaciones miembro de IPLF para resolver problemas provenientes de la selección de contenido (pertinencia), la facilidad de acceso a este (accesibilidad), la construcción de significado (inteligibilidad) y la aplicación del contenido (aplicabilidad). En particular, se hace hincapié en el principio de inteligibilidad para lograr la articulación de ideas complejas, por ser, además, el punto de intervención de traductores y correctores. Sin duda, quedan por explorar otras intervenciones posibles relativas a los diversos principios. 
En la sección 4, se abordan problemas de traducción y corrección que surgen tanto de la redacción en uno u otro idioma como de las diferencias idiosincráticas entre idiomas. Se propone la conceptualización de un sistema de revisión bidimensional que permite, por un lado, atender a cuestiones que atañen a la construcción de significado (textualidad) y, por otro, al estilo o registro (adecuación). En cuestiones de textualidad, se presentan técnicas referentes a la construcción de sentido dentro de la oración, para atender a la gramaticalidad y aceptabilidad, y entre oraciones, para llegar al texto en su totalidad, y atender, así, a la cohesión y la coherencia. A fin de abordar problemas de traducibilidad, se sugiere la domesticación del texto de llegada como recurso para superar diferencias idiosincráticas (de representación de la realidad) entre idiomas y ser objeto de revisión aplicando las técnicas de redacción antes mencionadas. En este aspecto, quedan por explorar diversas cuestiones, como la nominalización y el sistema de marcadores discursivos en tanto estrategias cohesivas o el tratamiento del sistema clítico, de gran riqueza en español (particularmente, el uso del "se" y de los clíticos dativos), entre muchas otras.

Para concluir, se observa que, aun cuando no se tenga injerencia sobre el texto de partida, quizás ya publicado, a menudo es posible desambiguar o corregir el texto de llegada.

\section{Referencias bibliográficas}

ADAMS, C.; CAMPBELL, A.; MONTGOMERY, R.; COOPER, M. y KIRKPATRICK, A. (eds.) (2021). Web Content Accessibility Guidelines (WCAG) 2.2. https://www.w3.org/TR/WCAG22/. Fecha de consulta: 5 de junio de 2021.

AMERICAN PSYCHOLOGICAL ASSOCIATION. (2020). Publication manual of the American Psychological Association, 7. ${ }^{a}$ ed. American Psychological Association.

ASD (2017). Simplified Technical English, Specification ASD-STE100. AeroSpace and Defence Industries Association of Europe. http://www.asd-ste100.org. Fecha de consulta: 5 de junio de 2021.

ASTM Standard F2575 (2014). Standard Guide for Quality Assurance in Translation. ASTM International. https://www.astm.org/Standards/F2575.htm. Fecha de consulta: 5 de junio de 2021.

AUTISM QUEBEC (2020). Fiches d'éducation financière.

http://autismequebec.org/fr/fiches-d-education-financiere/14. Fecha de consulta: 5 de junio de 2021.

BAJTÍN, M. (1999). Estética de la creación verbal, 10. ed. Madrid. Siglo XXI Editores.

BALMFORD, C. (2020). An ISO plain language standard. https://www.iplfederation.org/an-iso-plain-language-standard/. Fecha de consulta: 5 de junio de 2021.

BERGEN, B. y CHANG, N. (en preparación). Embodied Construction Grammar. https://cogsci.ucsd.edu/ bkbergen/papers/ECG_Handbook.pdf. Fecha de consulta: 5 de junio de 2021.

BRANSFORD, J. y JOHNSON, M. (1972). Contextual prerequisites for understanding: Some investigations of comprehension and recall. Journal of Verbal Learning and Verbal Behavior, vol. 11, 717-726.

BRATCHER HOSKINS, S. (1986). Text Superstructures. Journal of Reading, vol. 29(6), 538-543. 
CALVÈS, A. (2009). Empowerment: The History of a Key Concept in Contemporary Development Discourse. Revue Tiers Monde, vol. 4(4), 735-749.

CARTWRIGHT, M. (2020). Enrique V de Inglaterra. World History Encyclopedia. https://www.worldhistory.org/trans/es/1-18611/enrique-v-de-inglaterra/. Fecha de consulta: 5 de junio de 2021.

CHEEK, A. (2010). Defining plain language. Clarity, vol. 64, 5-15. https://clarity.wildapricot.org/Resources/Documents/64_032111_04_final.pdf. Fecha de consulta: 5 de junio de 2021.

CIAPUSCIO, G. (2005). La noción de género en la lingüística sistémico funcional y en la $\begin{array}{llll}\text { lingüística textual. Signos, } & \text { 38(57), }\end{array}$ http://www.scielo.cl/scielo.php?pid=S071809342005000100003\&script=sci_arttext. Fecha de consulta: 5 de junio de 2021.

CUTTS, M. (2013). Oxford Guide to Plain English, 4. ${ }^{\text {a }}$ ed. Oxford University Press.

DEPARTAMENTO ADMINISTRATIVO DE LA FUNCIÓN PÚBLICA (2011). Guía de lenguaje ciudadano para la Administración Pública Colombiana. Colombia. https://www.funcionpublica.gov.co/documents/418537/4754239/00337_0005 3_16.+Guia+de+Lenguaje+Ciudadano+para+la+Administracion+Publica+Colombi ana.pdf/47e84c12-b37a-4cfd-a18b-e47bd7e4a223?download=true. Fecha de consulta: 5 de junio de 2021.

DANEŠ, F. (1974). Functional sentence perspective and the organization of the text. En: DANEŠ, F. (ed.). Papers on Functional Sentence Perspective. Berlín-Boston. De Gruyter Mouton.

DECHTER, G. (2011). IRS Aims for Letter-Perfect Language. Center for American Progress.

https://www.americanprogress.org/issues/general/news/2011/05/16/9588/ir s-aims-for-letter-perfect-language/. Fecha de consulta: 5 de junio de 2021.

DIRECCIÓN NACIONAL DEL SISTEMA ARGENTINO DE INFORMACIÓN JURÍDICA (2019). Guía SAIJ de lenguaje claro. Ministerio de Justicia y Derechos Humanos. Argentina. http://lenguajeclaroargentina.gob.ar/guia-saij-de-lenguaje-claro/. Fecha de consulta: 5 de junio de 2021.

DIRECCIÓN NACIONAL DE IMPRESIONES Y PUBLICACIONES OFICIALES (2011). Programa de Lenguaje Ciudadano de IMPO. Uruguay. https://www.impo.com.uy/lenguajeciudadano/. Fecha de consulta: 5 de junio de 2021.

DEPARTAMENTO NACIONAL DE PLANEACIÓN (2015). Guía de lenguaje claro para servidores públicos de Colombia. Colombia. Imprenta Nacional. http://www.portaltributariodecolombia.com/wp-content/uploads/2015/07/ portaltributariodecolombia_guia-de-lenguaje-claro-para-servidores-publicos.pdf. Fecha de consulta: 5 de junio de 2021.

EGGINS, S. (2011). An Introduction to Systemic Functional Linguistics. Bloomsbery Academic.

ESTADO SIMPLE, COLOMBIA ÁGIL (2019). 25 preguntas frecuentes sobre la estrategia. https://www.colombiaagil.gov.co/preguntas-frecuentes/26-preguntasfrecuentes-sobre-estado-simple-colomb. Fecha de consulta: 5 de junio de 2021.

FERNÁNDEZ-ORDÓÑEZ, I. (s. f.). Alfonso X el Sabio en la historia del español. http://www.cervantesvirtual.com/obra-visor/alfonso-x-el-sabio-en-la-historiadel-espaol-0/html/023c114a-82b2-11df-acc7-002185ce6064_11.html. Fecha de consulta: 5 de junio de 2021. 
FERREIRO, S. (2012). Ley Fácil: Democratizando la Legislación. IFLA World Library and Information Congress. https://www.ifla.org/past-wlic/2012/214-ferreiro-es.pdf. Fecha de consulta: 5 de junio de 2021.

FLESCH, R. (1948). A New Readability Yardstick. Journal of Applied Psychology, vol. 32(3), 221-233.

FLESCH, R. (1949). The Art of Readable Writing. Harper and Row Publishers.

FLESCH, R. (1951). The Art of Clear Thinking. Harper and Brothers Publishing.

FUNCIÓN PÚBLICA. (2021, 22 de abril). Episodio 4: ¿Qué es el lenguaje claro? Podcast del Departamento Administrativo De La Función Pública. https://www.funcionpublica.gov.co/episodios//asset_publisher/nmfblu6ytxsr/content/episodio-4-\%257c$\% 25 \mathrm{c} 2 \% 25 \mathrm{bfqu} \% 25 \mathrm{c} 3 \% 25 \mathrm{a} 9$-es-el-lenguaje-claro-

GABALLO, V. (2012). Exploring the Boundaries of Transcreation in Specialized Translation. ESP Across Cultures, vol. 9, 95-113.

GARCÍA, O. (2020). El lenguaje claro aumenta la confianza en las empresas. https://www.bbva.com/es/el-lenguaje-claro-aumenta-la-confianza-en-lasempresas/. Fecha de consulta: 5 de junio de 2021.

GARNER, B. A. (2009). Garner on language and writing: Selected essays and speeches of Bryan Garner. American Bar Association.

GHIO, E. y FERNÁNDEZ, M. D. (2008). Lingüística sistémico funcional. Aplicaciones a la lengua española. Waldhuter Editores.

GIBA, J. (2014). Developing skills in scientific writing. Barcelona. Esteve Foundation. http://esteve.org/wp-content/uploads/2018/01/13066.pdf. Fecha de consulta: 5 de junio de 2021.

GIORA, R. (1985). Notes towards a Theory of Text Coherence. Poetics Today, vol. 6(4), 699.

HALLIDAY, M. A. K. y HASAN, R. (1976). Cohesion in English. Longman.

HARRIS, L.; KLEIMANN, S. y MOWAT, C. (2010). Setting plain language standards. $\begin{array}{lll}\text { Clarity, } & \text { vol. } & 64,\end{array}$ https://clarity.wildapricot.org/Resources/Documents/64_032111_04_final.pdf Fecha de consulta: 5 de junio de 2021.

HAYES, J. R. y FLOWER, L. S. (1980). Identifying the organization of writing processes. En: L. GREGG y E. STEINBERG (eds.) Cognitive processes in writing: An interdisciplinary approach. Erlbaum, 3-30.

HEALTHWISE (2020). Charlie Learns About Coronavirus: A Storybook for Kids. Healthwise. https://www.southwesternhealth.org/sites/default/files/202004/covid_charlie_storybook.pdf. Fecha de consulta: 5 de junio de 2021.

HOROWITZ, R. (1985). Text Patterns: Part I. Journal of Reading, vol. 28(5), 448-454. https://www.jstor.org/stable/i40001601. Fecha de consulta: 5 de junio de 2021.

HOWICK, J.; MITTOO, S.; ABEL, L.; HALPERN, J. y MERCER, S. W. (2020). A price tag on clinical empathy? Factors influencing its cost-effectiveness. Journal of the Royal Society of Medicine, vol. 113(10), 389-393. doi: 10.1177/0141076820945272.

IKEA IBÉRICA S. A. (2019). Informe no financiero. Año fiscal 2019. https://www.ikea.com/es/es/files/pdf/d6/ba/d6ba26cd/informe-no-financieroikea-fy19.pdf. Fecha de consulta: 5 de junio de 2021.

INTERNATIONAL PLAIN LANGUAGE FEDERATION (s .f.) Plain Language Definitions. https://www.iplfederation.org/plain-language/ Fecha de consulta: 5 de junio de 2021. 
ISO STANDARD 17100 (2015). Translation services - Requirements for translation services. ISO. https://www.iso.org/standard/59149.html. Fecha de consulta: 5 de junio de 2021.

ISO STANDARD 24495-1 (2015). Plain language - Part 1: Governing principles and guidelines. ISO. https://www.iso.org/standard/78907.html. Fecha de consulta: 5 de junio de 2021.

JAKOBSON, R. (1960). Closing Statement: Linguistics and Poetics. En: T. A. SEBEOK (ed.). Style in language. The Technology Press of Massachusetts Institute of Technology and John Wiley \& Sons.

JAMES, S.; SAWYER A. y WALLSCHUTZKY, I. (2015). Tax simplification: A review of initiatives in Australia, New Zealand and the United Kingdom. eJournal of Tax Research, vol. 13(1), 280-302.

JARRETT, C. y RIDISH, J. G. (2019). Readability Formulas: 7 Reasons to Avoid Them and What to Do Instead UXmatters. https://www.uxmatters.com/mt/archives/2019/07/readability-formulas-7reasons-to-avoid-them-and-what-to-do-instead.php. Fecha de consulta: 5 de junio de 2021.

KEARL, B. (1948). A Closer Look at Readability Formulas. Journalism Quarterly, vol. 25(4), 344-348. https://doi.org/10.1177/107769904802500403

KELLY, N. (2020). Creating a More Accessible Localization Definition. Born to be Global. https://borntobeglobal.com/2020/05/22/localization-definition/. Fecha de consulta: 5 de junio de 2021.

KIMBLE, J. (1992). Plain English: A charter for clear writing. Thomas M. Cooley Law Review, vol. 9(1), 1-58.

KIMBLE, J. (1996-1997). Writing for dollars, writing to please. The Scribes Journal of Legal Writing, vol. 6: 1-38.

KINCAID, J. P.; FISHBURNE, R. P. Jr; ROGERS, R. y CHISSOM, B. (1975). Derivation of new readability formulas (Automated Readability Index, Fog Count and Flesch Reading Ease Formula) for Navy enlisted personnel. Research Branch Report 8-75, Millington, TN: Naval Technical Training, U. S. Naval Air Station, Memphis, TN.

KLOBERDANZ, K. (2017). Honey, I Shrunk the Contract: How Plain English is Helping GE Keep its Business Humming. GE News. 2 de marzo. https://www.ge.com/news/reports/keep-simple-plain-english-helping-ge-keepbusiness-humming. Fecha de consulta: 5 de junio de 2021.

KRASHEN, S. (2012). Academic Jibberish. RELC Journal, vol. 43(2), 283-285. doi: $10.1177 / 0033688212453045$.

KRATHWOHL, D. R. (2002). A revision of Bloom's taxonomy: An overview. Theory into Practice, vol. 41(4), 212-218.

LAKOFF, G. y JOHNSON, M. (2003). Metaphors we live by. University of Chicago Press.

LAPOLLA, R. (2019). Arguments for Seeing Theme-Rheme and Topic-Comment as Separate Functional Structures. En J. R. MARTIN, Y. J. DORAN y G. FIGUEDERO (eds.). Systemic Functional Language Description: Making Meaning Matter. Routledge. doi: 10.4324/9781351184533-6.

LEGGETTE, H. R.; RUTHERFORD, T.; DUNSFORD, D. y COSTELLO, L. (2015). A Review and Evaluation of Prominent Theories of Writing. Journal of Applied Communications, vol. 99(3). doi: 10.4148/1051-0834.1056

LEIVADA, E. y WESTERGAARD. M. (2020). Acceptable Ungrammatical Sentences, Unacceptable Grammatical Sentences, and the Role of the Cognitive Parser. Frontiers in Psychology, vol. 11, 364. doi: 10.3389/fpsyg.2020.00364. 
Ley 26.653 de 2010. Ley de Accesibilidad de la Información en las Páginas Web en Argentina. http://servicios.infoleg.gob.ar/infolegInternet/anexos/175000179999/175694/norma.htm. Fecha de consulta: 5 de junio de 2021.

Ley 34/2002 de 2002. Ley de Servicios de la Sociedad de la Información y de Comercio Electrónico en

España. https://www.boe.es/boe/dias/2002/07/12/pdfs/A25388-25403.pdf. Fecha de consulta: 5 de junio de 2021.

Ley General para la Inclusión de las Personas con Discapacidad en México de 2011. http://www.diputados.gob.mx/LeyesBiblio/pdf/LGIPD_120718.pdf. Fecha de consulta: 5 de junio de 2021.

LINDERHOLM, T.; EVERSON, M. G.; VAN DEN BROEK, P.; MISCHINSKI, M.; CRITTENDEN, A. y SAMUELS, J. (2000). Effects of Causal Text Revisions on More- and Less-Skilled Readers' Comprehension of Easy and Difficult Texts. Cognition and Instruction, vol. 18(4), 525-556. doi: 10.1207/s1532690xci1804_4.

MAHER, C. (1998). “Well, here I am, Daniel, in the lion's den” [Chrissie Maher's speech to the European Commission.]

http://www.plainenglish.co.uk/campaigning/past-campaigns/european-

union/chrissie-maher-s-speech-to-the-european-commission.html. Fecha de consulta: 5 de junio de 2021.

MAHER, G. y WILD, J. (2002). Plain English Campaign, UK. Cuarto Congreso Bianual de Plain Language Association International.

http://en.copian.ca/library/research/plain2/campaign/campaign.pdf. Fecha de consulta: 5 de junio de 2021.

MACDONALD, D. (2015). How Miss America Changed Citibank, Part II. American Banker, 23 de enero. https://www.americanbanker.com/opinion/how-miss-americachanged-citibank-part-ii. Fecha de consulta: 5 de junio de 2021.

MACK, J. E.; MELTZER-ASSCHER, A.; BARBIERI, E. y THOMPSON, C. K. (2013). Neural correlates of processing passive sentences. Brain Sciences, vol. 3(3), 1198-1214. doi: 10.3390/brainsci3031198.

MARAZZATO SPARANO, R. (2016). Historia del español y su gramática ¡Al rescate del español! 28 de junio. https://rescatedelesp.com/historia-del-espanol-y-sugramatica/\#more-213. Fecha de consulta: 5 de junio de 2021.

MARAZZATO SPARANO, R. (2017). Lessons from the Plain Language Movement. Quincuagésimo octavo Congreso Anual de American Translators Association. Washington DC. EE. UU. https://web.atanet.org/conf/2017/byspecial/\#T-9. Fecha de consulta: 5 de junio de 2021.

MARAZZATO SPARANO, R. (2018). Translating Romance Languages into English. Quincuagésimo noveno Congreso Anual de American Translators Association. New Orleans. EE. UU. https://web.atanet.org/conf/2018/sessions/?track=18ATA-

ANNUAL-4914\&token=395015f5f9494b96299ca49666e04400. Fecha de consulta: 5 de junio de 2021.

MARAZZATO SPARANO, R. (2019). Plain Language 2.0 \& Health Literacy: New Strategies for Better Outcomes. Decimosegundo Congreso Bianual de Plain Language Association International. Oslo, Noruega. https://plainlanguagenetwork.org/conferences/2019-oslo-norway/. Fecha de consulta: 5 de junio de 2021.

MARAZZATO SPARANO, R. (2021a). New Writing Fundamentals. Manuscrito en preparación. 
MARAZZATO SPARANO, R. (2021b). Empatía en la corrección de textos: intervenciones tendientes a un lenguaje claro y libre de sesgo. VI Congreso Internacional de Correctores de Textos en Español.

MARTÍNEZ DE SOUSA, J. (1999). Manual de edición y autoedición. Madrid. Pirámide.

MAVERICK, M. (1944). Lengthy Memoranda and Gobbledygook Language. Be short and use Plain English. 24 de marzo. Smaller War Plants Corporation.

MAYLATH, B. y AMANT, M. (eds.) (2019). Translation and Localization: A Guide for Technical and Professional Communicators. ATTW Series in Technical and Professional Communication. Routledge.

MINISTERIO PARA LAS ADMINISTRACIONES PÚBLICAS DE ESPAÑA (1990). Manual de estilo del lenguaje administrativo. Madrid. Instituto Nacional de Administración Pública.

MITAMURA, T. y NYBERG E. (2001). Automatic Rewriting for Controlled Language Translation Language Technologies Institute. Carnegie Mellon University.

MONTOLÍO, E. y TASCÓN, M. (s. f.) El derecho a entender. Comunicación clara. Una herramienta moderna y eficaz para que la Administración y las empresas dialoguen con la ciudadanía de una forma clara y transparente. Prodigioso Volcán. https://www.prodigiosovolcan.com/pv/comunicacion/assets/ComunicacionClar a.pdf. Fecha de consulta: 5 de junio de 2021.

MULTIREGIONAL CLINICAL TRIALS (s. f.) Health Literacy in Clinical Research. https://health.gov/healthypeople/objectives-and-data/social-determinantshealth/literature-summaries/health-literacy. Fecha de consulta: 5 de junio de 2021.

MUNDAY, J. (2016). Introducing Translation Studies. Routledge.

MUÑOZ FERNANDEZ, A. (2018). Legible. https://legible.es/. Fecha de consulta: 5 de junio de 2021.

MURPHY, J. (2012). A Short History of Writing Instruction: From Ancient Greece to Contemporary America, 3. ${ }^{\mathrm{a}}$ ed. Routledge.

NADEAU, S. E. (2012). The neural architecture of grammar. MIT Press.

NIDA, E. (1969). Toward a Science of Translating. Brill.

NOMURA, M.; SKAT NIELSEN, G. y TRONBACKE, B. (eds.) (2012). Directrices para materiales de lectura fácil. The International Federation of Library Associations and Institutions.

O'HAYRE, J. (1966). Gobbledygook Has Gotta Go. Bureau of Land Management's Western Information Office.

http://training.fws.gov/history/HistoricDocuments.html. Fecha de consulta: 5 de junio de 2021.

OECD. (2016). Skills Matter: Further Results from the Survey of Adult Skills. OECD Skills Studies, OECD Publishing. https://www.oecd-ilibrary.org/education/skillsmatter_9789264258051-en. Fecha de consulta: 5 de junio de 2021.

ODPHP, Office of Disease Prevention and Health Promotion (2021). Health Literacy in Healthy People 2030. https://health.gov/our-work/national-healthinitiatives/healthy-people/healthy-people-2030/health-literacy-healthy-people2030. Fecha de consulta: 5 de junio de 2021.

ORGANIZACIÓN MUNDIAL DE LA SALUD. (2017). WHO Strategic Framework for Effective Communications.

https://cdn.who.int/media/docs/default-source/documents/communicationframework.pdf?sfvrsn=93aa6138_0 
OSSANDON, C. (2017). Transparencia, claridad y responsabilidad, tres pilares para lograr la confianza de los clientes. Comunicación TCR. https://www.bbva.com/es/transparencia-claridad-responsabilidad-tres-pilareslograr-confianza-clientes/. Fecha de consulta: 5 de junio de 2021.

PANOU, D. (2013). Equivalence in Translation Theories: A Critical Evaluation. Theory and Practice in Language Studies, vol. 3(1), 1-6. doi: 10.4304/tpls.3.1.1-6.

PÉREZ GARCÍA, J. M. y LÓPEZ DE LA CRUZ, L. (2015). La lectura fácil: una apuesta de valor para las organizaciones. Revista Española de Discapacidad, vol. 3 (1), 187-192. https://www.cedd.net/redis/index.php/redis/article/view/152/pdf_32. 9/2/16. Fecha de consulta: 5 de junio de 2021.

PIACENTE, T. (2014). El lenguaje escrito y los sistemas de escritura: Implicancias para su aprendizaje. Documento de trabajo. Facultad de Psicología de la Universidad Nacional de La Plata.

PINKER, S. (2014). The Sense of Style: The Thinking Person's Guide to Writing in the 21st Century. New York. Penguin.

PLAIN LANGUAGE ASSOCIATION INTERNATIONAL (s. f.) What is plain language? https://plainlanguagenetwork.org/plain-language/what-is-plain-language/.

Fecha de consulta: 5 de junio de 2021.

PLAIN WRITING ACT OF 2010. Pub.L. 111-274. https://www.govinfo.gov/content/pkg/PLAW-111publ274/pdf/PLAW111publ274.pdf. Fecha de consulta: 5 de junio de 2021.

POBLETE, C. A. y FUENZALIDA GONZÁLEZ, P. (2018). Una mirada al uso de lenguaje claro en el ámbito judicial latinoamericano. Revista de Llengua i Dret, vol. 69, 120138. http://urbeetius.org/wp-content/uploads/2020/08/poblete.pdf. Fecha de consulta: 5 de junio de 2021.

PODER JUDICIAL DE PERÚ (2014). Manual judicial de lenguaje claro y accesible a los ciudadanos. Fondo Editorial del Poder Judicial. https://www.pj.gob.pe/wps/wcm/connect/7b17ec0047a0dbf6ba8abfd87f5ca43 e/MANUAL+JUDICIAL+DE+LENGUAJE+CLARO+Y+ACCESIBLE.pdf?MOD=AJPERES . Fecha de consulta: 5 de junio de 2021.

PORTOLÉS, J. (1994). Algunos comentarios sobre la teoría de la pertinencia. Pragmalingüística, vol. 2, 407-431. http://rodin.uca.es/xmlui/bitstream/handle/10498/8728/18228550.pdf. Fecha de consulta: 5 de junio de 2021.

QUINTILIANO. Institutio Oratoria. 8.2.24. http://data.perseus.org/citations/urn:cts:latinLit:phi1002.phi0018.perseuslat1:2.24. Fecha de consulta: 5 de junio de 2021.

RAMÍREZ POLO, L. (2012). Los lenguajes controlados y la documentación técnica: mejorando la traducibilidad. Revista Tradumàtica. Tecnologies de la Traducció, vol. 10 , 192-204. https://ddd.uab.cat/pub/tradumatica/tradumatica_a2012n10/tradumatica_a201 2n10p192.pdf. Fecha de consulta: 5 de junio de 2021.

Reglamento 536/2014 del Parlamento Europeo, del 16 de abril de 2014, sobre los ensayos clínicos de medicamentos de uso humano, y por el que se deroga la Directiva 2001/20/CE.

https://ec.europa.eu/health/sites/health/files/files/eudralex/vol1/reg_2014_536/reg_2014_536_en.pdf. Fecha de consulta: 5 de junio de 2021.

Rehabilitation Act of 1973, Pub.L. 93-112, 87 Stat. 355, Sec. 508. Electronic and Information Technology. https://www.Section508.Gov/

REYES, A. (2018). La antigua retórica: la crítica en la edad ateniense. Fondo de Cultura. 
SISTEMA ARGENTINO DE INFORMACIÓN JURÍDICA (2019). Guía SAIJ de lenguaje claro. Pautas para redactar información jurídica en lenguaje claro. http://lenguajeclaroargentina.gob.ar/wpcontent/uploads/2019/08/Gu\%C3\%ADa-SAIJ-de-lenguaje-claro.pdf. Fecha de consulta: 5 de junio de 2021.

SÁNCHEZ TRIGO, E. (2017). Tipologías textuales y traducción. Revista de Traductología, vol., 121-133. doi: 10.24310/TRANS.2002.v0i6.2931.

SARLO, B. (2019). La lengua en disputa. Beatriz Sarlo y Santiago Kalinowski. Modera Cecilia Fanti [video]. YouTube. 22 de agosto.

https://www.youtube.com/watch?v=FVqopqV4XdM. Fecha de consulta: 5 de junio de 2021.

SCOTT, B. (s. f.). Readability Formulas. https://readabilityformulas.com/. Fecha de consulta: 5 de junio de 2021.

SCHRIVER, K. (2017). Plain Language in the US Gains Momentum: 1940-2015. IEEE Transactions on Professional Communication, vol. 60(4), 343-383. doi: 10.1109/TPC.2017.2765118

SCHRIVER, K. (1996). Dynamics in Document Design: Creating Texts for Readers. Wiley.

SECRETARÍA DE LA FUNCIÓN PÚBLICA (2007). Manual de lenguaje claro. México. https://www.economia.gob.mx/files/empleo/ManualLenguaje.pdf. Fecha de consulta: 5 de junio de 2021.

SLOBIN, D. (2004). The many ways to search for a frog: Linguistic typology and the expression of motion events. En: S. STRÖMQVIST y L. VERHOEVEN (eds.). Language in mind: Advances in the study of language and thought. MIT Press, 157-192.

STEPHENS, C. (ed.) (2010). Plain Language in Plain English. Plain Language Wizardry.

STEPHENS, C. y HARRISON-WHITESIDE, K. (2007). Plain Language in Organizations: An Action Plan. Book 2. Plain Language Wizardry.

TALMY, L. (1972). Semantic structures in English and Atsagewi. Doctoral Dissertation. University of California, Berkeley.

TALMY, L. (2000). Toward a cognitive semantics. Volume II: Typology and process in concept structuring. Cambridge. MIT Press.

VAN DIJK, T. (1980). Macrostructures. An Interdisciplinary Study of Global Structures in Discourse, Interaction, and Cognition. Lawrence Erlbaum Associates.

VENUTI, L. (2004). The translator's invisibility: A history of translation. Routledge.

VINAY, J.-P. y DARBELNET, J. (1977). Stylistique comparée du français et de l'anglais. Paris: Didier. (Primera edición de 1958).

VOGELZANG, M.; THIEL, C. M.; ROSEMANN, S.; RIEGER J. W. y RUIGENDIJK, E. (2020). Neural Mechanisms Underlying the Processing of Complex Sentences: An fMRI Study. Neurobiology of Language, vol. 1 (2), 226-248. doi: 10.1162/nol_a_00011

W3C (2005). Introducción a las pautas de accesibilidad para el contenido web. https://www.w3.org/WAI/standards-guidelines/wcag/es. Fecha de consulta: 5 de junio de 2021.

WILLIAMS, J. (1990). Style: Toward Clarity and Grace. The University of Chicago Press.

ZAWOZNIK, M. (2021). Aplicación de normas académicas en textos de especialidad y decisiones editoriales en publicaciones científicas. VI Congreso Internacional de Correctores de Textos en Español. 